\title{
Molecular characterization of urban organic aerosol in tropical India: contributions of primary emissions and secondary photooxidation
}

\author{
P. Q. Fu' ${ }^{1}$, K. Kawamura ${ }^{1}$, C. M. Pavuluri ${ }^{1}$, T. Swaminathan ${ }^{2}$, and J. Chen ${ }^{3,4,1}$ \\ ${ }^{1}$ Institute of Low Temperature Science, Hokkaido University, Sapporo, 060-0819, Japan \\ ${ }^{2}$ Department of Chemical Engineering, Indian Institute of Technology Madras, Chennai, 600036, India \\ ${ }^{3}$ State Key Laboratory of Environmental Geochemistry, Institute of Geochemistry, Chinese Academy of Sciences, \\ Guiyang, 550002, China \\ ${ }^{4}$ Graduate School of the Chinese Academy of Sciences, Beijing, 100039, China
}

Received: 12 August 2009 - Published in Atmos. Chem. Phys. Discuss.: 15 October 2009

Revised: 19 February 2010 - Accepted: 11 March 2010 - Published: 19 March 2010

\begin{abstract}
Organic molecular composition of $\mathrm{PM}_{10}$ samples, collected at Chennai in tropical India, was studied using capillary gas chromatography/mass spectrometry. Fourteen organic compound classes were detected in the aerosols, including aliphatic lipids, sugar compounds, lignin products, terpenoid biomarkers, sterols, aromatic acids, hydroxy/polyacids, phthalate esters, hopanes, Polycyclic Aromatic Hydrocarbons (PAHs), and photooxidation products from biogenic Volatile Organic Compounds (VOCs). At daytime, phthalate esters were found to be the most abundant compound class; however, at nighttime, fatty acids were the dominant one. Di-(2-ethylhexyl) phthalate, $\mathrm{C}_{16}$ fatty acid, and levoglucosan were identified as the most abundant single compounds. The nighttime maxima of most organics in the aerosols indicate a land/sea breeze effect in tropical India, although some other factors such as local emissions and long-range transport may also influence the composition of organic aerosols. However, biogenic VOC oxidation products (e.g., 2-methyltetrols, pinic acid, 3-hydroxyglutaric acid and $\beta$-caryophyllinic acid) showed diurnal patterns with daytime maxima. Interestingly, terephthalic acid was maximized at nighttime, which is different from those of phthalic and isophthalic acids. A positive relation was found between 1,3,5-triphenylbenzene (a tracer for plastic burning) and terephthalic acid, suggesting that the field burning
\end{abstract}

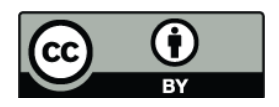

Correspondence to: K. Kawamura (kawamura@lowtem.hokudai.ac.jp) of municipal solid wastes including plastics is a significant source of terephthalic acid. Organic compounds were further categorized into several groups to clarify their sources. Fossil fuel combustion (24-43\%) was recognized as the most significant source for the total identified compounds, followed by plastic emission (16-33\%), secondary oxidation (8.6$23 \%$ ), and microbial/marine sources $(7.2-17 \%)$. In contrast, the contributions of terrestrial plant waxes $(5.9-11 \%)$ and biomass burning (4.2-6.4\%) were relatively small. This study demonstrates that, in addition to fossil fuel combustion and biomass burning, the open-burning of plastics in urban area also contributes to the organic aerosols in South Asia.

\section{Introduction}

Primary organic aerosol (POA, particle mass directly emitted from sources such as plant material, soil dust, biomass and fossil fuel burning) and secondary organic aerosol (SOA, particle mass formed by the oxidation of gas-phase precursors in the atmosphere) are ubiquitous in the atmosphere (e.g., Robinson et al., 2007; de Gouw and Jimenez, 2009; Hallquist et al., 2009). These organics account for up to $70 \%$ of the fine aerosol mass, and potentially control the physicochemical properties of atmospheric particles (Kanakidou et al., 2005). Organic aerosols are highlighted for the past decade because they are important environmental issues related to global and regional climate, chemistry of the atmosphere,

Published by Copernicus Publications on behalf of the European Geosciences Union. 
biogeochemical cycling, and people's health (Crutzen and Andreae, 1990; Kanakidou et al., 2005; Pöschl, 2005; Andreae and Rosenfeld, 2008).

India has experienced serious air pollution problem due to its rapid economic growth and urbanization in the past decade. It is regarded as a major source region of the Indo-Asian haze, due to significant industrial emissions, coal burning, vehicular exhaust emission, and waste incineration (Lelieveld et al., 2001). Indo-Asian haze is also known as Atmospheric Brown Clouds (ABCs), which consist of a persistent and large-scale layer of air pollutants containing a mixture of Black Carbon (BC), Organic Carbon (OC), and dust. ABCs significantly absorb and scatter solar radiation (Lelieveld et al., 2001; Ramanathan et al., 2005; Seinfeld, 2008; Szidat, 2009), and thus impact on South Asian climate and hydrological cycle (Ramanathan et al., 2005). Efforts to reduce the extent of $\mathrm{ABCs}$ require the knowledge of their composition and sources (Szidat, 2009).

Biomass/biofuel (including wood, agricultural residues, and dried animal manure) burning and fossil fuel combustion are considered as the major sources of carbonaceous aerosols in this region (Lelieveld et al., 2001; Venkataraman et al., 2005; Stone et al., 2007). Recently, Gustafsson et al. (2009) used radiocarbon $\left({ }^{14} \mathrm{C}\right)$ as a tracer to quantify biomass and fossil fuel contributions to the ABCs. Chowdhury et al. (2007) reported the organic speciation and source apportionment of fine particles in four Indian cities using a receptor-based method. Furthermore, it is important to understand the changes in the organic aerosol composition due to photochemical oxidation at a molecular level (Robinson et al., 2006; Rudich et al., 2007). Tropical region may provide a unique site to study the photochemical aging of organic aerosols because of the high ambient temperature and strong sunlight irradiation. However, knowledge about the organic molecular composition of atmospheric aerosols in tropical India is still limited.

In this study, we present the concentrations of 14 compound classes (174 organic species) in tropical Indian aerosols, including aliphatic hydrocarbons, sugar compounds, lignin and resin products, aromatic acids, polyacids, plastic emission products, hopanes, PAHs, as well as SOA tracers from the photooxidation of biogenic volatile organic compounds (BVOCs) such as isoprene, $\alpha / \beta$-pinene and $\beta$-caryophyllene. Based on the molecular distributions, their possible sources and seasonal/diurnal differences are reported. Contributions of each compound class to OC and Water-Soluble Organic Carbon (WSOC) in the samples are also discussed. As far as we are aware, this is one of the most comprehensive studies of ambient organic aerosols at a molecular level. Our work adds to the increasing database on the chemical characterizations of organic aerosols in the atmosphere.

\section{Experimental}

\subsection{Aerosol sampling}

Day- and night-time $\mathrm{PM}_{10}$ samples were collected on the campus of the Indian Institute of Technology Madras (IITM), Chennai $\left(13.04^{\circ} \mathrm{N} ; 80.17^{\circ} \mathrm{E}\right)$, tropical India during winter (23 January-6 February, $n=29$ ) and summer (23-31 May, $n=20)$ 2007. IITM campus is located in a natural forest area, and is ca. $3 \mathrm{~km}$ away from the coast. An air sampler was set up on the rooftop of the Mechanical Sciences building (18 $\mathrm{m}$ a.g.l. - above ground level). Sampling was conducted during daytime (06:00-18:00 LT - local time) and nighttime (18:00-06:00 LT) using a high volume air sampler (Envirotech APM $460 \mathrm{DX}$, India) and pre-combusted $\left(450^{\circ} \mathrm{C}\right.$, $4 \mathrm{~h})$ quartz fiber filters (Pallflex 2500QAT-UP, $20 \times 25 \mathrm{~cm}$ ). The sample was placed in a pre-combusted glass jar with a Teflon-lined screw cap and stored in a dark freezer room at $-20^{\circ} \mathrm{C}$ prior to analysis. It should be noted that the sampling artefacts might occur due to the evaporation/adsorption processes of semi-volatile organics on the filter or particle surface during sampling. However, we consider that the evaporative loss of semi-volatile organics should be minimal due to the following reasons.

If the evaporative loss were significant, we could detect a serious molecular fractionation among different molecular weight ranges. First, Low Molecular Weight (LMW) $n$-alkanes are more volatile than high molecular weight $n$ alkanes. If the evaporative loss of $n$-alkanes were significant due to the temperature effect, then the ratios of LMW $n$-alkanes to HMW $n$-alkanes should be higher in winter than in summer. However, lower ratios of LMW $n$-alkanes $\left(\mathrm{C}_{18}-\right.$ $\left.\mathrm{C}_{24}\right)$ to HMW $n$-alkanes $\left(\mathrm{C}_{25}-\mathrm{C}_{40}\right)$ were detected in winter $(0.22 \pm 0.27)$ than summer $(0.26 \pm 0.23)$. This does not support a serious evaporative loss of semi-volatile $n$-alkanes. Second, phthalate esters that are well known as semi-volatile organic pollutants in the atmosphere showed higher concentrations in summer (295-857 $\mathrm{ng} \mathrm{m}^{-3}$, average $553 \mathrm{ng} \mathrm{m}^{-3}$ ) than in winter $\left(175-598 \mathrm{ng} \mathrm{m}^{-3}\right.$, average $\left.303 \mathrm{ng} \mathrm{m}^{-3}\right)$. This again denies a potential importance of the artefacts. Third, three- and four-ring PAHs (LMW-PAHs) are more volatile than those of seven- and eight-ring PAHs (HMW-PAHs). The ratios of LMW-PAHs/HMW-PAHs in the present study were $0.16 \pm 0.07$ in winter versus $0.18 \pm 0.05$ in summer. This similarity does not support a significant fractionation during sampling, suggesting that evaporative losses are negligible for different rings of PAHs. Therefore, we feel that our sampling technique does not have serious sampling artefacts.

However, some semi-volatile compounds may be lost from the aerosol particles on the filter. Thus we think that the reported concentrations may still be underestimated. It is important to note that there are a large number of documented studies that focused on the similar semi-volatile organics using high-volume air samplers to collect atmospheric aerosols at different geographical locations in the world including 
tropics,... (e.g., Simoneit et al., 1990; Kawamura and Sakaguchi, 1999; Simoneit and Elias, 2000; Alves et al., 2001; Graham et al., 2002; Wang et al., 2006a).

\subsection{Extraction, derivatization, and GC/MS determination}

Detailed analytical method has been described elsewhere (Fu et al., 2008, 2009b). Briefly, filter aliquots were extracted with dichloromethane/methanol $(2: 1, \mathrm{v} / \mathrm{v})$, followed by concentration, and derivatization with $50 \mu \mathrm{l}$ of N,O-bis-(trimethylsilyl)trifluoroacetamide (BSTFA) with $1 \%$ trimethylsilyl chloride and $10 \mu \mathrm{l}$ of pyridine at $70^{\circ} \mathrm{C}$ for $3 \mathrm{~h}$. After reaction, the derivatives were diluted by the addition of $140 \mu \mathrm{l}$ of $n$-hexane with $1.43 \mathrm{ng} \mathrm{\mu l}^{-1}$ of the internal standard ( $\mathrm{C}_{13} n$-alkane) prior to GC/MS injection. GC/MS analyses of the samples were performed on a HewlettPackard model 6890 GC coupled to a Hewlett-Packard model 5973 Mass-Selective Detector (MSD). The GC was equipped with a split/splitless injector and a DB-5MS fused silica capillary column $(30 \mathrm{~m} \times 0.25 \mathrm{~mm}$ i.d., $0.25 \mu \mathrm{m}$ film thickness). The mass spectrometer was operated on Electron Ionization (EI) mode at $70 \mathrm{eV}$ and scanned from 50 to $650 \mathrm{Da}$. Data were acquired and processed with the Chemstation software. GC/MS response factors were determined using authentic standards. Recoveries of the quantified organic compounds were in general better than $80 \%$. Field blank filters were treated as real samples for quality assurance. The results showed contamination levels are less than $5 \%$ of real samples for any species detected. The data reported here were corrected for the field blanks but not for recoveries.

OC was determined using a Sunset Lab EC/OC Analyzer following the Interagency Monitoring of Protected Visual Environments (IMPROVE) thermal evolution protocol. An aliquot of each filter was also analyzed for WSOC. The filter aliquot was first extracted with $10 \mathrm{ml}$ of organic-free pure water by ultrasonication for $10 \mathrm{~min}$. The water extracts were then filtered using a pre-rinsed syringe filter (Millex-GV with $0.22 \mu \mathrm{m}$ pore size, Millipore). WSOC in the water extracts was measured using a Shimadzu TOC-5000A (Pavuluri et al., 2010).

\section{Results and discussion}

\subsection{Meteorology and air mass back trajectories}

Detailed weather information has been mentioned elsewhere (Pavuluri et al., 2010). Briefly, the weather in Chennai is generally hot and humid. Ambient temperatures during the campaigns varied from $14.2-34.9^{\circ} \mathrm{C}$ (average $23^{\circ} \mathrm{C}$ ) in winter and $28.3-41^{\circ} \mathrm{C}\left(32^{\circ} \mathrm{C}\right)$ in summer. No rain was recorded during sampling. A clear diurnal oscillation in wind speed and wind direction was found in Chennai due to a strong land-sea thermal gradient. The onset of sea breeze at daytime that introduce cool marine air passing over a warm land surface results in a Thermal Internal Boundary Layer (TIBL) below the Planetary Boundary Layer (PBL). In contrast, the onset of land breeze at nighttime may remove the TIBL. The PBL moves down at night.

Ten-day air mass trajectory analysis (HYSPLIT, NOAA) showed that most of the air masses were transported long distances from North India and the Middle East in early winter (23-28 January) and from Southeast Asia over the Bay of Bengal in late winter (29 January-6 February). In contrast, the Arabian Sea, Indian Ocean and South Indian continent are suggested as major source regions in summer (2231 May) (Pavuluri et al., 2010). Back trajectory analysis also showed that the air masses originated from mixed regions (North India and Southeast Asia) between 30 January and 2 February. Following the air mass trajectory analysis, we present the seasonal differences of some compound classes and their discussions as three categories of air mass transport patterns.

\subsection{Speciation of particulate organic compounds}

Homologous of 14 organic compound classes, i.e., $n$-alkanes, fatty acids, fatty alcohols, anhydrosugars, sugars/sugar alcohols, lignin products, terpenoid biomarkers, sterols, aromatic acids, phthalate esters, hopanes, Polycyclic Aromatic Hydrocarbons (PAHs), hydroxy-/polyacids, and biogenic SOA tracers (e.g., 2-methyltetrols and pinic acid) were detected in the tropical Indian aerosols. Table 1 presents the concentrations of more than 170 organic compounds detected in this study. Concentrations of total quantified organic compounds in the tropical Indian aerosols were higher in summer $\left(650-3325 \mathrm{ng} \mathrm{m}^{-3}\right.$, average $\left.1683 \mathrm{ng} \mathrm{m}^{-3}\right)$ than in winter (380-2461 ng m$\left.{ }^{-3}, 1192 \mathrm{ng} \mathrm{m}^{-3}\right)$. Among the organic compounds detected, fatty acids and phthalates are the major compound classes, followed by $n$-alkanes and anhydrosugars. Other compound classes are relatively minor. Figure 1 presents a typical GC/MS trace (Total Ion Current, TIC) for the samples. As a single compound detected (on average), di-(2-ethylhexyl) phthalate (DEHP) was the most abundant both in summer and in winter, followed by levoglucosan, Diisobutyl Phthalate (DiBP), and $\mathrm{C}_{16: 0}$ fatty acid in winter (see Fig. A1 for chemical structures). However in summer, levoglucosan became less abundant than $\mathrm{C}_{16: 0}$ fatty acid and DiBP.

\subsection{Day-/nighttime variations in the concentrations: effect of land/sea breeze}

As shown in Figs. 2 and 3, concentrations of most organic compound classes are more abundant at nighttime than at daytime, except for the secondary oxidation products such as biogenic SOA tracers. For example, $n$-alkanes, fatty acids, fatty alcohols, terpenoid biomarkers, and sterols showed 


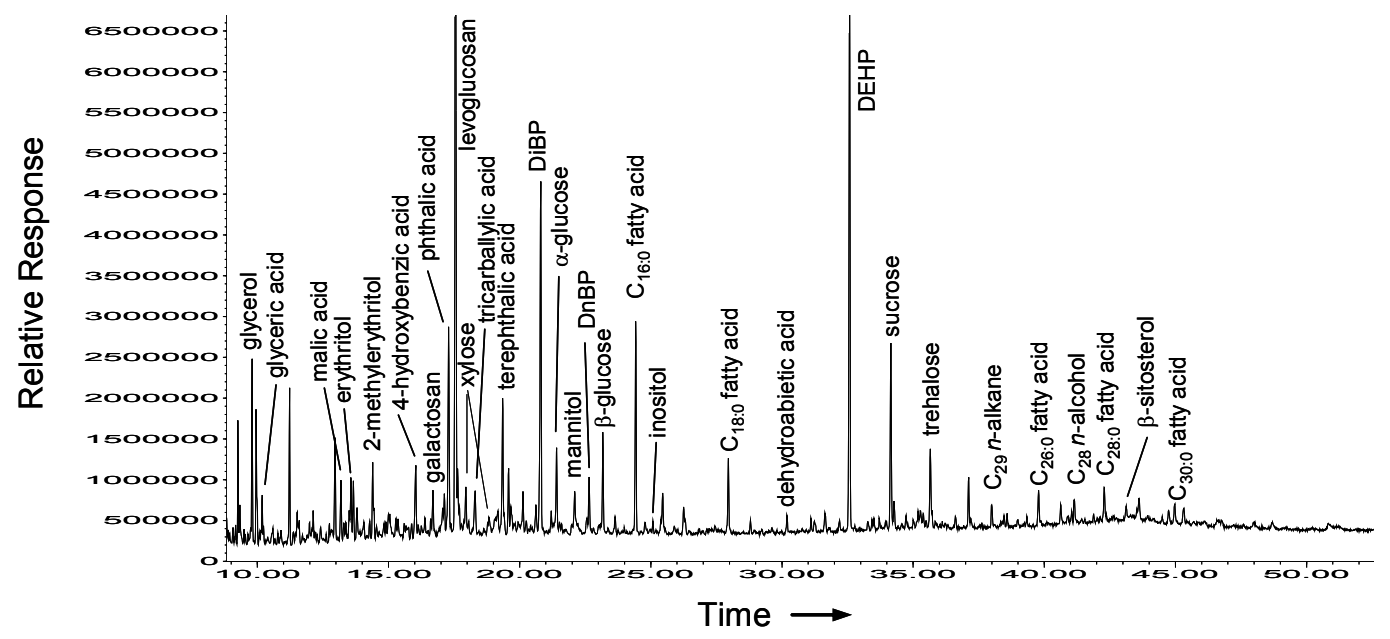

Fig. 1. A typical GC/MS trace (Total Ion Current: TIC) for a total extract (TMS derivatized) of aerosol particles from tropical India. For the abbreviation, see Table 1.

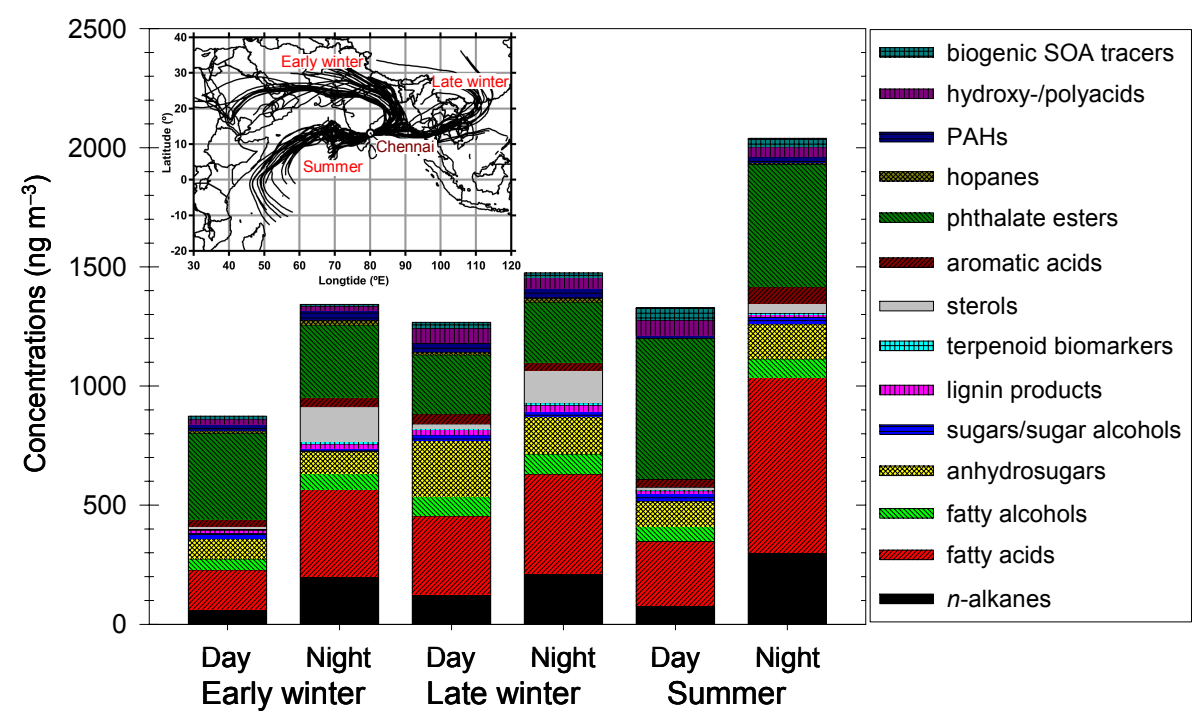

Fig. 2. Chemical compositions of organic compounds in atmospheric aerosols from Chennai, tropical India. Ten-day air mass back trajectories arriving at $500 \mathrm{~m}$ above Chennai $\left(13.04^{\circ} \mathrm{N}\right.$ and $80.17^{\circ} \mathrm{E}$ ) in winter (23 January to 6 February) and summer (22-31 May) 2007, showed three major pathways reflecting early winter, late winter and summer.

clear nighttime maxima. The nighttime maxima may be caused by the land breeze that transport terrestrial organic matter from the inland to Chennai (Pavuluri et al., 2010). During the sampling period in the present study, the wind was southwesterly (land-breeze) during early hours of the day. However, it turned northeasterly and southeasterly at noon hours (12:00-13:00 LT) during both winter and summer, and accelerated subsequently as a result of the onset of sea breeze. The sea breeze could continue until late night (Venkatesan et al., 2009) but its strength varied with the changes in wind speed.

During late winter, the differences between day- and nighttime concentrations of the compound classes were relatively minor. This is reasonable because the land/sea breeze effect was less important during late winter when the air masses originated from Southeast Asia over the Bay of Bengal. As seen in Fig. 2, anhydrosugars (levoglucosan and its two isomers, galactosan (II) and mannosan (III), the tracers for biomass burning (Simoneit, 2002), showed higher daytime concentrations (average $235 \mathrm{ng} \mathrm{m}^{-3}$ ) than nighttime $\left(156 \mathrm{ng} \mathrm{m}^{-3}\right)$ in late winter. The higher concentrations are associated with sea breeze at daytime. This demonstrates that biomass-burning activities in Southeast Asia are very active and the atmospheric transport of smoke aerosols from this region is more significant than the local emissions in tropical India at nighttime during late winter. 

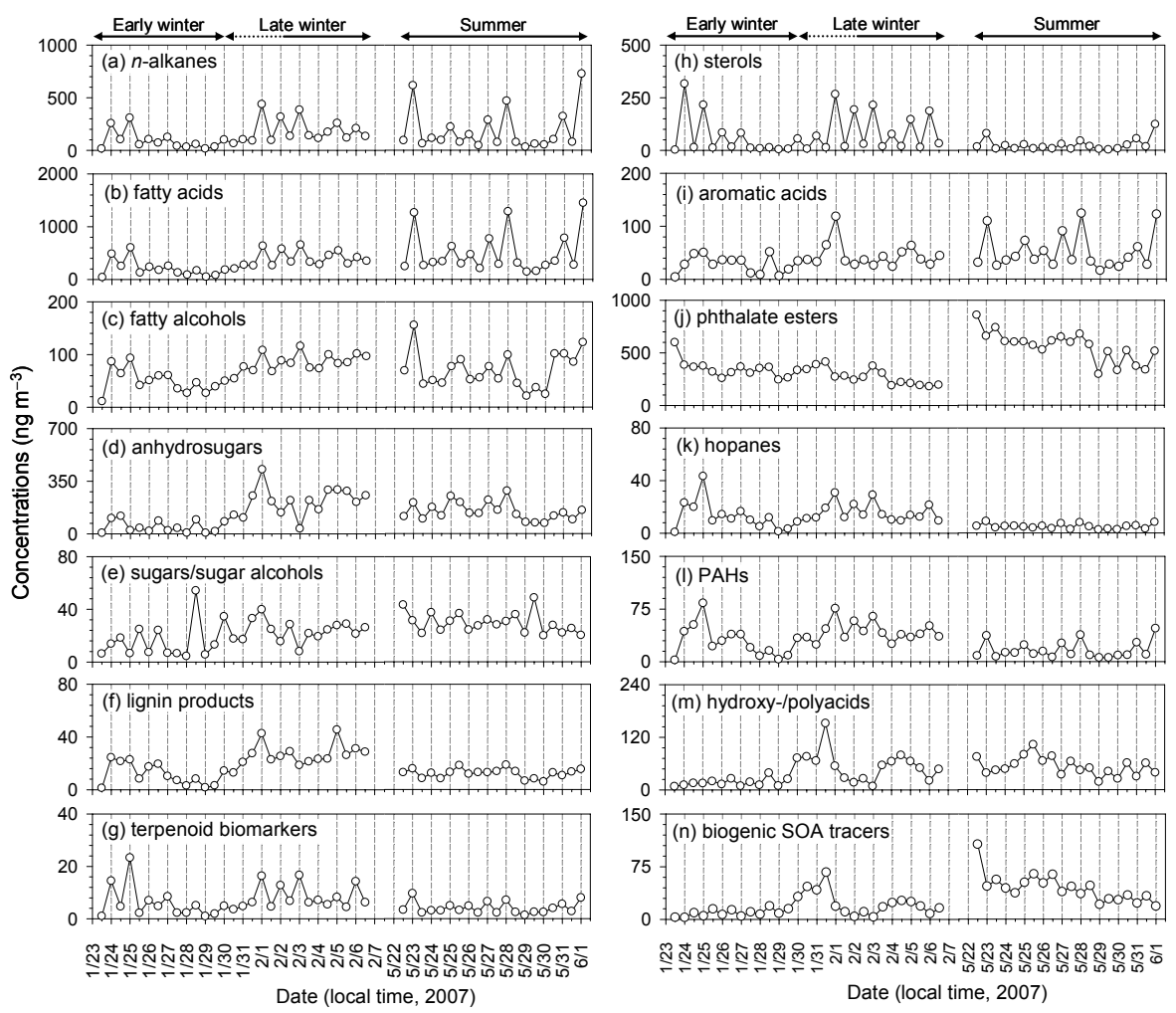

Fig. 3. Temporal variations in the concentrations of aliphatic lipids and other organic compound classes detected in the tropical Indian aerosols.

\subsection{Biomass burning tracers}

Levoglucosan, formed during the pyrolysis of cellulose, has been detected in urban (Wang et al., 2006a, b; Zheng et al., 2006; Chowdhury et al., 2007; Yttri et al., 2007), marine (Simoneit and Elias, 2000; Simoneit et al., 2004; Wang et al., 2009), and the polar regions (Stohl et al., 2007; Fu et al., 2009a). In this study, levoglucosan was found to be one of the most abundant species among the individually identified compounds with a concentration range of 50.7-213 $\mathrm{ng} \mathrm{m}^{-3}$ (average $111 \mathrm{ng} \mathrm{m}^{-3}$ ) in summer (Table 1), which are similar to those reported in Delhi $\left(210 \pm 40 \mathrm{ng} \mathrm{m}^{-3}\right)$ and Kolkata aerosols ( $75 \pm 15 \mathrm{ng} \mathrm{m}^{-3}$ ) (Chowdhury et al., 2007). On the other hand, the concentrations of levoglucosan in winter ranged from 4.30 to $361 \mathrm{ng} \mathrm{m}^{-3}\left(112 \mathrm{ng} \mathrm{m}^{-3}\right)$, which are much lower than those reported in Delhi $\left(5300 \pm 1100 \mathrm{ng} \mathrm{m}^{-3}\right)$, Kolkata $\left(5500 \pm 1100 \mathrm{ng} \mathrm{m}^{-3}\right)$, and Mumbai aerosols $\left(910 \pm 180 \mathrm{ng} \mathrm{m}^{-3}\right)$ (Chowdhury et al., 2007). However, our results are 1-2 orders of magnitude higher than those reported in the Indian Ocean aerosols collected at the Maldives climate observatories in Gan (12.3$25.4 \mathrm{ng} \mathrm{m}^{-3}$ ) and Kanimaadhoo (1.97-8.62 $\mathrm{ng} \mathrm{m}^{-3}$ ) sites in the ABC monitoring network (Stone et al., 2007). Interestingly, no correlation $\left(R^{2}=0.12\right)$ was found between the concentrations of levoglucosan and non-sea-salt $\mathrm{K}^{+}$(nss-K) (Pavuluri et al., unpublished data) in all the samples. This indicates that nss- $\mathrm{K}$ is not a good biomass-burning tracer at least in Chennai. We consider that cow-dung burning is an important source of levoglucosan in tropical India. The burning of cow-dung is not an "emitter" of nss-K. However, the correlation coefficient $\left(R^{2}\right)$ between levoglucosan and nss-K was 0.36 for winter samples $(n=29)$ versus 0.10 for summer samples $(n=20)$. Such a weak positive correlation found in winter was supported by the fact that the air masses mainly originated from North India and Southeast Asia. In North India, the burning of wood for heating is common in winter.

Seven lignin products (vanillin, shonanin, and 3hydroxybenzoic, 4-hydroxybenzoic, 3,4-dihydroxybenzoic, vanillic, and syringic acids) were detected in the aerosols with a total concentration of $19.3 \pm 11.2 \mathrm{ng} \mathrm{m}^{-3}$ in winter versus $12.3 \pm 3.49 \mathrm{ng} \mathrm{m}^{-3}$ in summer (Fig. 3f). 4Hydroxybenzoic acid (IV) was the dominant species in both winter and summer, followed by 3,4-dihydroxybenzoic acid. 3,4-Dihydroxybenzoic acid (protocatechuic acid) is an antioxidant that contains a polyphenolic structure. In human health, polyphenol antioxidants are thought to be instrumental in combating oxidative stress, a process associated with some neurodegenerative diseases and some cardiovascular diseases (http://en.wikipedia.org/wiki/Polyphenol_ antioxidant). These hydroxy acids have been reported in continental aerosols (Fu et al., 2008) and smoke particles (Oros and Simoneit, 2001; Simoneit, 2002). Vanillic acid (V) is 
Table 1. Concentrations of organic compounds detected in the atmospheric aerosols from Chennai, tropical India $\left(\mathrm{ng} \mathrm{m}^{-3}\right)$.

\begin{tabular}{|c|c|c|c|c|c|c|c|c|}
\hline \multirow[t]{2}{*}{ Compounds } & \multicolumn{4}{|c|}{ Winter $(n=29)$} & \multicolumn{4}{|c|}{ Summer $(n=20)$} \\
\hline & $\min$ & $\max$ & average & $\mathrm{SD}^{\mathrm{a}}$ & $\min$ & $\max$ & average & SD \\
\hline \multicolumn{9}{|l|}{ I. $n$-Alkanes } \\
\hline $\mathrm{C}_{18}$ & 0.07 & 0.85 & 0.46 & 0.20 & 0.07 & 2.15 & 0.55 & 0.49 \\
\hline $\mathrm{C}_{19}$ & 0.42 & 2.12 & 1.15 & 0.46 & 0.17 & 4.41 & 1.66 & 1.05 \\
\hline $\mathrm{C}_{20}$ & 0.40 & 3.26 & 1.45 & 0.71 & 0.06 & 5.67 & 1.34 & 1.36 \\
\hline $\mathrm{C}_{21}$ & 0.84 & 7.89 & 3.50 & 1.84 & 1.10 & 32.1 & 8.37 & 9.12 \\
\hline $\mathrm{C}_{22}$ & 0.29 & 7.34 & 2.25 & 1.88 & 0.39 & 4.63 & 2.04 & 1.31 \\
\hline $\mathrm{C}_{23}$ & 0.60 & 15.2 & 4.02 & 3.87 & 1.25 & 11.9 & 3.99 & 2.64 \\
\hline $\mathrm{C}_{24}$ & 0.59 & 26.7 & 6.13 & 6.81 & 1.34 & 21.6 & 5.72 & 5.05 \\
\hline $\mathrm{C}_{25}$ & 0.71 & 39.3 & 10.4 & 9.59 & 2.67 & 41.9 & 11.8 & 10.9 \\
\hline $\mathrm{C}_{26}$ & 0.48 & 37.1 & 11.0 & 9.59 & 2.25 & 46.8 & 13.0 & 13.3 \\
\hline $\mathrm{C}_{27}$ & 0.98 & 41.3 & 13.4 & 10.3 & 2.89 & 64.8 & 16.8 & 17.1 \\
\hline $\mathrm{C}_{28}$ & 0.59 & 31.9 & 10.5 & 8.76 & 1.84 & 53.4 & 13.7 & 15.4 \\
\hline $\mathrm{C}_{29}$ & 0.94 & 40.2 & 15.7 & 10.1 & 3.02 & 64.3 & 18.3 & 18.1 \\
\hline $\mathrm{C}_{30}$ & 0.24 & 25.6 & 8.93 & 7.09 & 1.48 & 49.8 & 12.2 & 14.3 \\
\hline $\mathrm{C}_{31}$ & 1.03 & 34.2 & 13.1 & 8.40 & 2.24 & 63.7 & 16.4 & 18.1 \\
\hline $\mathrm{C}_{32}$ & 0.22 & 22.6 & 7.68 & 6.37 & 1.10 & 49.5 & 11.1 & 13.8 \\
\hline $\mathrm{C}_{33}$ & 0.20 & 27.1 & 10.0 & 7.11 & 1.42 & 52.2 & 14.0 & 15.8 \\
\hline $\mathrm{C}_{34}$ & 0.05 & 17.6 & 5.13 & 4.79 & 0.70 & 37.3 & 8.58 & 11.0 \\
\hline $\mathrm{C}_{35}$ & 0.15 & 14.2 & 4.80 & 3.88 & 0.71 & 36.5 & 7.54 & 10.1 \\
\hline $\mathrm{C}_{36}$ & n.d. ${ }^{b}$ & 12.4 & 4.02 & 3.56 & 0.18 & 32.8 & 6.90 & 9.15 \\
\hline $\mathrm{C}_{37}$ & n.d. & 16.6 & 3.23 & 3.80 & 0.25 & 43.5 & 7.39 & 12.3 \\
\hline $\mathrm{C}_{38}$ & n.d. & 8.23 & 2.06 & 2.38 & n.d. & 23.0 & 3.91 & 6.32 \\
\hline $\mathrm{C}_{39}$ & n.d. & 7.32 & 1.81 & 2.35 & n.d. & 10.4 & 1.46 & 2.71 \\
\hline $\mathrm{C}_{40}$ & n.d. & 4.01 & 0.68 & 1.30 & n.d. & 5.56 & 0.65 & 1.36 \\
\hline subtotal & 15.2 & 437 & 141 & 110 & 30.9 & 727 & 187 & 200 \\
\hline $\mathrm{CPI}\left(\mathrm{C}_{21}-\mathrm{C}_{40}\right)^{\mathrm{c}}$ & 1.17 & 2.34 & 1.56 & 0.30 & 1.20 & 1.97 & 1.52 & 0.23 \\
\hline \multicolumn{9}{|c|}{ Plant Wax $n$-Alkanes ${ }^{\mathrm{d}}$} \\
\hline $\mathrm{C}_{23}$ & 0 & 0.62 & 0.12 & 0.15 & 0 & 0.76 & 0.28 & 0.28 \\
\hline $\mathrm{C}_{25}$ & 0 & 7.38 & 1.79 & 1.58 & 0 & 7.67 & 2.42 & 2.00 \\
\hline $\mathrm{C}_{27}$ & 0.32 & 6.77 & 2.59 & 1.68 & 0.79 & 14.7 & 3.40 & 3.08 \\
\hline $\mathrm{C}_{29}$ & 0.52 & 11.4 & 6.02 & 2.56 & 1.33 & 12.7 & 5.36 & 3.31 \\
\hline $\mathrm{C}_{31}$ & 0.80 & 10.1 & 4.79 & 2.02 & 0.95 & 23.3 & 4.81 & 4.86 \\
\hline $\mathrm{C}_{33}$ & 0.06 & 7.50 & 3.59 & 2.06 & 0.52 & 14.8 & 4.23 & 3.67 \\
\hline $\mathrm{C}_{35}$ & 0 & 3.04 & 0.42 & 0.67 & 0 & 1.49 & 0.20 & 0.40 \\
\hline $\mathrm{C}_{37}$ & 0 & 6.84 & 0.50 & 1.31 & 0 & 15.6 & 2.09 & 4.83 \\
\hline subtotal & 2.26 & 49.6 & 19.8 & 9.67 & 4.85 & 76.3 & 22.8 & 19.8 \\
\hline \multicolumn{9}{|l|}{ II. Fatty Acids } \\
\hline $\mathrm{C}_{8: 0}$ & 0.54 & 2.40 & 1.02 & 0.45 & 0.86 & 2.43 & 1.58 & 0.41 \\
\hline $\mathrm{C}_{9: 0}$ & 0.19 & 2.05 & 0.74 & 0.38 & 0.86 & 12.1 & 3.40 & 3.39 \\
\hline $\mathrm{C}_{10: 0}$ & 0.11 & 0.79 & 0.46 & 0.14 & 0.47 & 1.84 & 0.97 & 0.45 \\
\hline $\mathrm{C}_{11: 0}$ & n.d. & 0.90 & 0.34 & 0.25 & 0.01 & 1.99 & 0.67 & 0.50 \\
\hline $\mathrm{C}_{12: 0}$ & 1.64 & 9.14 & 3.63 & 1.90 & 3.26 & 19.6 & 9.08 & 5.81 \\
\hline $\mathrm{C}_{13: 0}$ & 0.18 & 1.35 & 0.66 & 0.31 & 0.56 & 2.18 & 1.15 & 0.43 \\
\hline $\mathrm{C}_{14: 0}$ & 1.85 & 21.4 & 8.00 & 4.40 & 7.80 & 86.4 & 24.6 & 18.6 \\
\hline $\mathrm{C}_{15: 0}$ & 0.38 & 4.67 & 2.38 & 0.99 & 3.29 & 14.7 & 6.53 & 3.25 \\
\hline $\mathrm{C}_{16: 0}$ & 6.45 & 217 & 90.9 & 63.4 & 77.7 & 780 & 275 & 218 \\
\hline $\mathrm{C}_{17: 0}$ & 0.29 & 5.27 & 2.54 & 1.51 & 1.27 & 14.6 & 5.19 & 4.03 \\
\hline $\mathrm{C}_{18: 0}$ & 2.04 & 110 & 43.7 & 33.2 & 14.4 & 287 & 85.4 & 83.9 \\
\hline $\mathrm{C}_{19: 0}$ & 0.01 & 3.31 & 1.46 & 0.92 & 0.49 & 6.53 & 2.22 & 1.85 \\
\hline $\mathrm{C}_{20: 0}$ & 0.45 & 14.6 & 6.49 & 4.44 & 1.27 & 24.5 & 7.31 & 7.26 \\
\hline $\mathrm{C}_{21: 0}$ & 0.18 & 4.18 & 1.98 & 1.00 & 0.45 & 6.70 & 2.34 & 1.90 \\
\hline $\mathrm{C}_{22: 0}$ & 0.72 & 29.6 & 12.6 & 7.75 & 1.84 & 28.7 & 9.38 & 8.64 \\
\hline $\mathrm{C}_{23: 0}$ & 0.45 & 10.7 & 6.08 & 2.54 & 0.87 & 14.4 & 5.09 & 4.21 \\
\hline $\mathrm{C}_{24: 0}$ & 1.51 & 49.5 & 23.3 & 11.8 & 3.02 & 36.5 & 13.9 & 9.61 \\
\hline
\end{tabular}


Table 1. Continued.

\begin{tabular}{|c|c|c|c|c|c|c|c|c|}
\hline \multirow[t]{2}{*}{ Compounds } & \multicolumn{4}{|c|}{ Winter $(n=29)$} & \multicolumn{4}{|c|}{ Summer $(n=20)$} \\
\hline & $\min$ & $\max$ & average & $\mathrm{SD}$ & $\min$ & $\max$ & average & SD \\
\hline $\mathrm{C}_{25: 0}$ & 0.60 & 9.25 & 5.41 & 1.93 & 0.75 & 12.2 & 3.93 & 3.26 \\
\hline $\mathrm{C}_{26: 0}$ & 1.56 & 32.6 & 19.8 & 7.87 & 2.11 & 27.6 & 10.4 & 6.77 \\
\hline $\mathrm{C}_{27: 0}$ & 0.58 & 6.87 & 4.14 & 1.39 & 0.47 & 9.49 & 2.89 & 2.48 \\
\hline $\mathrm{C}_{28: 0}$ & 1.31 & 25.4 & 15.4 & 5.31 & 2.21 & 22.9 & 10.1 & 5.40 \\
\hline $\mathrm{C}_{29: 0}$ & 0.42 & 6.08 & 3.79 & 1.26 & 0.50 & 6.26 & 2.55 & 1.79 \\
\hline $\mathrm{C}_{30: 0}$ & 1.52 & 19.1 & 11.4 & 3.43 & 1.61 & 13.2 & 6.82 & 3.19 \\
\hline $\mathrm{C}_{31: 0}$ & 0.06 & 3.12 & 1.63 & 0.74 & 0.14 & 4.55 & 1.34 & 1.16 \\
\hline $\mathrm{C}_{32: 0}$ & 0.15 & 8.74 & 5.36 & 1.83 & 0.74 & 6.34 & 2.88 & 1.34 \\
\hline $\mathrm{C}_{33: 0}$ & n.d. & 1.13 & 0.47 & 0.36 & n.d. & 1.29 & 0.47 & 0.33 \\
\hline $\mathrm{C}_{34: 0}$ & n.d. & 2.67 & 1.30 & 0.76 & 0.42 & 2.54 & 1.27 & 0.58 \\
\hline $\mathrm{C}_{16: 1}$ & n.d. & 2.03 & 0.25 & 0.45 & n.d. & 1.85 & 0.12 & 0.42 \\
\hline $\mathrm{C}_{18: 1}$ & 0.13 & 116 & 22.4 & 35.2 & n.d. & 74.8 & 7.19 & 16.8 \\
\hline $\mathrm{C}_{18: 2}$ & n.d. & 17.0 & 3.29 & 5.30 & n.d. & 2.67 & 0.17 & 0.61 \\
\hline $\mathrm{C}_{20: 1}$ & n.d. & 3.01 & 0.63 & 1.08 & n.d. & n.d. & n.d. & n.d. \\
\hline $\mathrm{C}_{22: 1}$ & n.d. & 3.34 & 0.45 & 0.93 & n.d. & n.d. & n.d. & n.d. \\
\hline subtotal & 40.5 & 657 & 302 & 179 & 141 & 1448 & 504 & 400 \\
\hline $\operatorname{CPI}\left(\mathrm{C}_{20: 0}-\mathrm{C}_{34: 0}\right)^{\mathrm{c}}$ & 3.11 & 6.40 & 3.99 & 0.84 & 2.80 & 5.09 & 3.61 & 0.56 \\
\hline \multicolumn{9}{|l|}{ III. Fatty Alcohols } \\
\hline $\mathrm{C}_{14}$ & n.d. & 0.95 & 0.27 & 0.23 & n.d. & 6.96 & 1.51 & 1.67 \\
\hline $\mathrm{C}_{15}$ & n.d. & 0.64 & 0.19 & 0.15 & 0.03 & 1.32 & 0.33 & 0.29 \\
\hline $\mathrm{C}_{16}$ & 0.10 & 4.18 & 1.72 & 1.05 & 0.50 & 6.86 & 2.58 & 1.53 \\
\hline $\mathrm{C}_{17}$ & 0.03 & 0.67 & 0.24 & 0.16 & 0.25 & 1.35 & 0.72 & 0.31 \\
\hline $\mathrm{C}_{18}$ & 0.24 & 3.04 & 1.10 & 0.67 & 0.98 & 13.9 & 3.58 & 3.03 \\
\hline $\mathrm{C}_{19}$ & 0.01 & 3.22 & 1.15 & 1.04 & 0.31 & 13.1 & 3.21 & 3.57 \\
\hline $\mathrm{C}_{20}$ & n.d. & 3.20 & 1.12 & 0.67 & 0.38 & 4.34 & 1.46 & 1.06 \\
\hline $\mathrm{C}_{21}$ & 0.01 & 1.79 & 0.58 & 0.43 & 0.02 & 3.40 & 0.87 & 0.81 \\
\hline $\mathrm{C}_{22}$ & 0.04 & 3.30 & 1.62 & 0.93 & 0.07 & 4.36 & 1.67 & 1.34 \\
\hline $\mathrm{C}_{23}$ & 0.24 & 1.92 & 0.95 & 0.48 & 0.02 & 4.55 & 1.15 & 1.24 \\
\hline $\mathrm{C}_{24}$ & 0.26 & 4.79 & 2.65 & 1.31 & 0.14 & 10.8 & 2.68 & 2.60 \\
\hline $\mathrm{C}_{25}$ & 0.05 & 2.22 & 0.93 & 0.68 & 0.08 & 7.97 & 1.55 & 1.98 \\
\hline $\mathrm{C}_{26}$ & 0.78 & 10.6 & 6.24 & 2.47 & 0.36 & 10.2 & 3.85 & 2.29 \\
\hline $\mathrm{C}_{27}$ & 0.07 & 3.44 & 1.26 & 0.86 & 0.03 & 5.90 & 1.49 & 1.62 \\
\hline $\mathrm{C}_{28}$ & 3.82 & 32.5 & 18.9 & 7.82 & 4.21 & 30.1 & 13.4 & 6.59 \\
\hline $\mathrm{C}_{29}$ & 0.24 & 3.20 & 1.37 & 0.79 & 0.23 & 8.88 & 1.43 & 1.92 \\
\hline $\mathrm{C}_{30}$ & 1.73 & 30.7 & 17.9 & 7.42 & 4.69 & 49.7 & 20.3 & 11.1 \\
\hline $\mathrm{C}_{31}$ & n.d. & 1.26 & 0.57 & 0.29 & 0.04 & 0.80 & 0.40 & 0.18 \\
\hline $\mathrm{C}_{32}$ & n.d. & 14.8 & 7.81 & 3.17 & 2.53 & 15.2 & 7.21 & 3.37 \\
\hline $\mathrm{C}_{33}$ & n.d. & 1.41 & 0.35 & 0.36 & n.d. & 1.04 & 0.28 & 0.30 \\
\hline $\mathrm{C}_{34}$ & n.d. & 3.57 & 0.92 & 0.97 & n.d. & 2.13 & 0.67 & 0.51 \\
\hline subtotal & 11.0 & 116 & 67.9 & 26.6 & 21.0 & 155 & 70.4 & 34.2 \\
\hline $\mathrm{CPI}\left(\mathrm{C}_{20}-\mathrm{C}_{34}\right)^{\mathrm{c}}$ & 4.68 & 16.8 & 9.75 & 2.94 & 3.34 & 27.7 & 10.9 & 6.77 \\
\hline \multicolumn{9}{|l|}{ IV. Anhydrosugars } \\
\hline galactosan & 0.35 & 23.7 & 8.07 & 6.22 & 2.71 & 10.8 & 5.84 & 2.12 \\
\hline mannosan & 0.26 & 42.6 & 13.4 & 12.0 & 3.75 & 20.3 & 9.90 & 4.52 \\
\hline levoglucosan & 4.30 & 361 & 112 & 95.4 & 50.7 & 213 & 111 & 45.2 \\
\hline subtotal & 4.99 & 427 & 134 & 112 & 59.6 & 244 & 127 & 51.6 \\
\hline \multicolumn{9}{|c|}{ V. Sugars/Sugar Alcohols } \\
\hline glycerol & 1.13 & 7.34 & 2.59 & 1.38 & 2.83 & 18.7 & 5.49 & 3.47 \\
\hline erythritol & 0.28 & 6.99 & 2.74 & 1.88 & 1.40 & 5.20 & 3.20 & 0.94 \\
\hline xylose & 0.32 & 9.03 & 3.13 & 2.13 & 1.80 & 6.81 & 3.91 & 1.49 \\
\hline arabitol & 0.16 & 2.49 & 1.05 & 0.67 & 0.78 & 4.06 & 1.51 & 0.70 \\
\hline fructose & 0.16 & 4.98 & 1.51 & 1.14 & 0.69 & 2.72 & 1.53 & 0.61 \\
\hline glucose & 0.49 & 20.6 & 3.86 & 4.00 & 2.61 & 14.8 & 6.82 & 3.24 \\
\hline mannitol & 0.06 & 2.38 & 0.54 & 0.52 & 0.82 & 5.56 & 2.20 & 1.11 \\
\hline inositol & 0.03 & 1.37 & 0.48 & 0.30 & 0.30 & 0.87 & 0.53 & 0.15 \\
\hline sucrose & 0.29 & 13.8 & 2.06 & 2.68 & 0.83 & 7.65 & 2.23 & 1.54 \\
\hline
\end{tabular}


Table 1. Continued.

\begin{tabular}{|c|c|c|c|c|c|c|c|c|}
\hline \multirow[t]{2}{*}{ Compounds } & \multicolumn{4}{|c|}{ Winter $(n=29)$} & \multicolumn{4}{|c|}{ Summer $(n=20)$} \\
\hline & $\min$ & $\max$ & average & SD & $\min$ & $\max$ & average & SD \\
\hline maltose & 0.01 & 7.27 & 0.81 & 1.40 & 0.44 & 3.14 & 1.08 & 0.58 \\
\hline trehalose & 0.06 & 2.66 & 1.22 & 0.81 & 0.34 & 1.63 & 1.00 & 0.33 \\
\hline subtotal & 4.39 & 54.2 & 20.0 & 11.8 & 19.9 & 48.8 & 29.5 & 7.83 \\
\hline \multicolumn{9}{|l|}{ VI. Lignin Products } \\
\hline 3-hydroxybenzoic acid & 0.14 & 2.24 & 0.86 & 0.43 & 0.46 & 3.33 & 1.11 & 0.85 \\
\hline 4-hydroxybenzoic acid & 0.49 & 29.5 & 9.93 & 6.58 & 2.46 & 9.60 & 5.29 & 1.93 \\
\hline 3,4-dihydroxybenzoic acid & 0.11 & 7.80 & 3.58 & 2.46 & 0.86 & 9.04 & 3.85 & 2.33 \\
\hline vanillin & 0.10 & 3.97 & 1.35 & 0.90 & 0.23 & 1.15 & 0.56 & 0.19 \\
\hline vanillic acid & 0.04 & 2.28 & 1.00 & 0.62 & 0.33 & 1.08 & 0.79 & 0.20 \\
\hline syringic acid & 0.03 & 4.21 & 2.07 & 1.27 & 0.24 & 1.13 & 0.62 & 0.25 \\
\hline shonanin & 0.02 & 1.26 & 0.54 & 0.38 & n.d. & 0.27 & 0.09 & 0.08 \\
\hline subtotal & 1.18 & 45.4 & 19.3 & 11.2 & 5.85 & 18.6 & 12.3 & 3.49 \\
\hline \multicolumn{9}{|l|}{ VII. Terpenoid Biomarkers } \\
\hline pimaric acid & n.d. & 0.59 & 0.15 & 0.18 & 0.01 & 0.13 & 0.05 & 0.03 \\
\hline isopimaric acid & n.d. & 1.24 & 0.26 & 0.34 & n.d. & 0.19 & 0.05 & 0.04 \\
\hline dehydroabietic acid & 0.58 & 14.2 & 4.30 & 3.16 & 0.76 & 5.53 & 2.56 & 1.35 \\
\hline abietic acid & n.d. & 0.64 & 0.13 & 0.18 & 0.01 & 0.05 & 0.02 & 0.01 \\
\hline 7-oxodehydroabietic acid & 0.15 & 1.16 & 0.38 & 0.28 & 0.09 & 0.59 & 0.19 & 0.10 \\
\hline$\alpha+\beta$-amyrin & 0.07 & 5.96 & 1.93 & 1.50 & 0.34 & 3.60 & 1.22 & 0.86 \\
\hline subtotal & 0.98 & 23.3 & 7.16 & 5.37 & 1.32 & 9.64 & 4.09 & 2.24 \\
\hline \multicolumn{9}{|l|}{ VIII. Sterols } \\
\hline cholesterol & 0.30 & 17.5 & 5.30 & 5.31 & 0.11 & 29.1 & 5.06 & 7.89 \\
\hline stigmasterol & 0.34 & 26.0 & 7.34 & 8.25 & n.d. & 12.4 & 2.48 & 3.57 \\
\hline$\beta$-sitosterol & 0.42 & 156 & 42.3 & 50.1 & 1.70 & 77.8 & 17.3 & 18.6 \\
\hline subtotal & 1.55 & 195 & 55.0 & 63.2 & 2.14 & 119 & 24.8 & 29.3 \\
\hline \multicolumn{9}{|l|}{ IX. Aromatic Acids } \\
\hline benzoic acid & 0.54 & 2.16 & 1.25 & 0.39 & 0.95 & 2.56 & 1.44 & 0.40 \\
\hline$o$-toluic acid & 0.00 & 0.30 & 0.16 & 0.07 & 0.04 & 0.40 & 0.15 & 0.09 \\
\hline$m$-toluic acid & 0.07 & 0.38 & 0.22 & 0.08 & 0.13 & 0.35 & 0.22 & 0.07 \\
\hline$p$-toluic acid & 0.06 & 0.53 & 0.24 & 0.10 & 0.08 & 0.34 & 0.22 & 0.08 \\
\hline phthalic acid & 0.42 & 31.2 & 5.79 & 5.87 & 1.92 & 11.5 & 5.82 & 2.81 \\
\hline isophthalic acid & 0.11 & 2.47 & 0.75 & 0.51 & 0.18 & 0.78 & 0.50 & 0.17 \\
\hline terephthalic acid & 1.61 & 111 & 28.1 & 21.0 & 11.8 & 117 & 43.4 & 34.8 \\
\hline subtotal & 3.90 & 118 & 36.5 & 21.9 & 15.9 & 124 & 51.8 & 33.9 \\
\hline \multicolumn{9}{|l|}{ X. Hydroxy-/Polyacids } \\
\hline glycolic acid & 4.03 & 50.5 & 16.3 & 10.6 & 5.30 & 29.7 & 14.1 & 6.08 \\
\hline salicylic acid & 0.11 & 1.07 & 0.38 & 0.22 & 0.15 & 0.42 & 0.27 & 0.07 \\
\hline glyceric acid & 0.15 & 7.88 & 2.51 & 2.34 & 1.91 & 9.85 & 5.69 & 2.00 \\
\hline malic acid & 1.00 & 36.9 & 9.29 & 8.97 & 7.74 & 44.8 & 24.0 & 10.1 \\
\hline tartaric acid & 0.02 & 13.4 & 2.52 & 3.42 & 1.98 & 13.0 & 5.66 & 3.33 \\
\hline citric acid & 0.06 & 3.68 & 0.74 & 0.84 & 0.14 & 2.35 & 0.92 & 0.62 \\
\hline tricarballylic acid & 0.35 & 39.1 & 6.33 & 8.28 & 0.78 & 4.91 & 2.45 & 1.06 \\
\hline subtotal & 7.83 & 152 & 38.1 & 32.2 & 18.1 & 103 & 53.1 & 21.0 \\
\hline \multicolumn{9}{|l|}{ XI. Biogenic SOA Tracers } \\
\hline 2-methylglyceric acid & 0.16 & 1.90 & 0.67 & 0.45 & 1.06 & 8.23 & 3.22 & 1.79 \\
\hline $\mathrm{C}_{5}$-alkene triols $\mathrm{s}^{\mathrm{e}}$ & 0.08 & 11.5 & 2.18 & 2.93 & 0.71 & 27.1 & 7.63 & 6.33 \\
\hline 2-methyltetrols & 0.17 & 10.7 & 2.75 & 2.78 & 3.54 & 43.2 & 13.9 & 9.10 \\
\hline$\Sigma$ isoprene SOA tracers & 0.45 & 22.4 & 5.60 & 5.93 & 5.74 & 78.6 & 24.7 & 16.2 \\
\hline $\begin{array}{l}\text { 3-hydroxyglutaric acid } \\
\text { (3-HGA) }\end{array}$ & 0.59 & 30.3 & 6.24 & 6.68 & 2.80 & 18.1 & 9.04 & 3.96 \\
\hline cis-pinonic acid & 0.10 & 1.69 & 0.82 & 0.36 & 1.49 & 14.2 & 4.31 & 3.23 \\
\hline
\end{tabular}


Table 1. Continued.

\begin{tabular}{|c|c|c|c|c|c|c|c|c|}
\hline \multirow[t]{2}{*}{ Compounds } & \multicolumn{4}{|c|}{ Winter $(n=29)$} & \multicolumn{4}{|c|}{ Summer $(n=20)$} \\
\hline & $\min$ & $\max$ & average & SD & $\min$ & $\max$ & average & SD \\
\hline cis-norpinic acid & 0.00 & 0.39 & 0.16 & 0.11 & 0.00 & 0.59 & 0.28 & 0.16 \\
\hline pinic acid & 0.14 & 3.14 & 1.00 & 0.82 & 0.67 & 3.78 & 2.08 & 0.80 \\
\hline MBTCA $^{f}$ & 0.00 & 1.27 & 0.25 & 0.30 & 0.19 & 1.32 & 0.76 & 0.35 \\
\hline$\Sigma \alpha / \beta$-pinene SOA tracers & 1.24 & 36.2 & 8.46 & 7.86 & 5.42 & 26.5 & 16.5 & 5.61 \\
\hline$\beta$-caryophyllinic acid & 0.41 & 9.52 & 2.76 & 1.92 & 1.01 & 5.44 & 2.69 & 0.98 \\
\hline subtotal & 2.69 & 66.9 & 16.8 & 14.7 & 18.3 & 107 & 43.9 & 20.0 \\
\hline \multicolumn{9}{|l|}{ XII. Plasticizers/Antioxidants } \\
\hline dimethyl phthalate (DMP) & n.d. & 0.12 & 0.04 & 0.04 & 0.04 & 0.16 & 0.09 & 0.03 \\
\hline diethyl phthalate (DEP) & 0.32 & 23.0 & 4.00 & 5.05 & 0.26 & 15.8 & 5.03 & 4.54 \\
\hline diisobutyl phthalate (DiBP) & 13.5 & 373 & 104 & 66.2 & 7.44 & 294 & 127 & 68.7 \\
\hline di- $n$-butyl phthalate (DnBP) & 6.80 & 103 & 24.9 & 17.8 & 10.5 & 211 & 42.4 & 44.7 \\
\hline $\begin{array}{l}\text { di-(2-ethylhexyl) phthalates } \\
\text { (DEHP) }\end{array}$ & 96.2 & 270 & 171 & 43.5 & 177 & 579 & 379 & 118 \\
\hline subtotal of phthalates & 175 & 598 & 303 & 89.0 & 295 & 857 & 553 & 140 \\
\hline $\begin{array}{l}\text { tris(2,4-di-tert-butyl-phenyl) } \\
\text { phosphateg }\end{array}$ & n.d. & 1.46 & 0.33 & 0.30 & n.d. & 1.54 & 0.42 & 0.48 \\
\hline \multicolumn{9}{|l|}{ XIII. Hopanes } \\
\hline $\mathrm{C}_{27} \alpha$ & n.d. & 1.36 & 0.39 & 0.36 & n.d. & 0.32 & 0.08 & 0.08 \\
\hline $\mathrm{C}_{27} \beta$ & n.d. & 0.18 & 0.05 & 0.06 & n.d. & 0.11 & 0.01 & 0.03 \\
\hline $\mathrm{C}_{29} \alpha \beta$ & 0.41 & 8.22 & 2.97 & 1.76 & 0.33 & 1.45 & 0.92 & 0.30 \\
\hline $\mathrm{C}_{29} \beta \alpha$ & n.d. & 1.04 & 0.27 & 0.24 & n.d. & 0.22 & 0.08 & 0.07 \\
\hline $\mathrm{C}_{30} \alpha \beta$ & 0.26 & 5.38 & 1.93 & 1.13 & 0.31 & 1.13 & 0.65 & 0.22 \\
\hline $\mathrm{C}_{30} \beta \alpha$ & n.d. & 0.59 & 0.11 & 0.13 & n.d. & 0.28 & 0.05 & 0.06 \\
\hline $\mathrm{C}_{31} \alpha \beta \mathrm{S}$ & n.d. & 3.95 & 1.52 & 0.89 & 0.21 & 1.86 & 0.64 & 0.43 \\
\hline $\mathrm{C}_{31} \alpha \beta \mathrm{R}$ & n.d. & 4.14 & 1.32 & 0.88 & 0.17 & 1.29 & 0.47 & 0.27 \\
\hline $\mathrm{C}_{31} \beta \alpha$ & n.d. & 0.94 & 0.24 & 0.24 & n.d. & 0.39 & 0.06 & 0.10 \\
\hline $\mathrm{C}_{32} \alpha \beta \mathrm{S}$ & n.d. & 3.20 & 1.25 & 0.77 & 0.17 & 1.06 & 0.50 & 0.26 \\
\hline $\mathrm{C}_{32} \alpha \beta \mathrm{R}$ & n.d. & 2.95 & 0.97 & 0.67 & 0.15 & 0.58 & 0.34 & 0.13 \\
\hline $\mathrm{C}_{33} \alpha \beta \mathrm{S}$ & n.d. & 2.50 & 0.80 & 0.56 & 0.10 & 0.67 & 0.29 & 0.13 \\
\hline $\mathrm{C}_{33} \alpha \beta \mathrm{R}$ & n.d. & 1.78 & 0.52 & 0.37 & 0.09 & 0.41 & 0.23 & 0.09 \\
\hline $\mathrm{C}_{34} \alpha \beta \mathrm{S}$ & n.d. & 2.28 & 0.67 & 0.50 & n.d. & 0.48 & 0.25 & 0.13 \\
\hline $\mathrm{C}_{34} \alpha \beta \mathrm{R}$ & n.d. & 2.05 & 0.52 & 0.43 & n.d. & 0.27 & 0.16 & 0.09 \\
\hline $\mathrm{C}_{35} \alpha \beta \mathrm{S}$ & n.d. & 1.95 & 0.51 & 0.42 & n.d. & 0.55 & 0.14 & 0.16 \\
\hline $\mathrm{C}_{35} \alpha \beta \mathrm{R}$ & n.d. & 1.56 & 0.38 & 0.32 & n.d. & 0.27 & 0.09 & 0.08 \\
\hline subtotal & 0.75 & 43.2 & 14.4 & 9.09 & 2.47 & 8.88 & 4.97 & 1.91 \\
\hline \multicolumn{9}{|l|}{ XIV. PAHs } \\
\hline 1,2,4-triphenylbenzene & n.d. & 0.68 & 0.20 & 0.18 & 0.08 & 1.24 & 0.31 & 0.33 \\
\hline 1,3,5-triphenylbenzene & 0.08 & 15.4 & 4.24 & 3.54 & 0.78 & 38.7 & 9.22 & 11.3 \\
\hline phenanthrene & 0.01 & 0.60 & 0.20 & 0.14 & 0.01 & 0.15 & 0.05 & 0.03 \\
\hline anthracene & n.d. & 0.16 & 0.05 & 0.04 & n.d. & 0.06 & 0.02 & 0.01 \\
\hline fluoranthene & 0.19 & 1.57 & 0.72 & 0.32 & 0.12 & 0.24 & 0.18 & 0.03 \\
\hline pyrene & 0.18 & 1.69 & 0.81 & 0.35 & 0.19 & 0.38 & 0.27 & 0.06 \\
\hline benzo(b)fluorine & 0.01 & 0.59 & 0.20 & 0.13 & n.d. & 0.15 & 0.06 & 0.05 \\
\hline benz(a)anthracene & 0.07 & 2.51 & 0.97 & 0.58 & 0.12 & 0.33 & 0.20 & 0.05 \\
\hline chrysene/triphenylene & 0.20 & 2.83 & 1.16 & 0.64 & 0.20 & 0.52 & 0.31 & 0.09 \\
\hline benzo(b)fluoranthene & 0.39 & 12.7 & 6.36 & 3.28 & 0.73 & 2.20 & 1.34 & 0.41 \\
\hline benzo(k)fluoranthene & 0.20 & 3.78 & 1.98 & 0.94 & 0.28 & 0.50 & 0.40 & 0.07 \\
\hline benzo(e)pyrene & 0.20 & 4.66 & 2.24 & 1.13 & 0.30 & 0.93 & 0.56 & 0.18 \\
\hline benzo(a)pyrene & 0.17 & 6.85 & 3.04 & 1.79 & 0.31 & 0.86 & 0.54 & 0.14 \\
\hline perylene & 0.01 & 1.70 & 0.74 & 0.45 & 0.11 & 0.24 & 0.16 & 0.04 \\
\hline indeno( $1,2,3$-cd)pyrene & 0.13 & 7.89 & 2.86 & 2.18 & 0.47 & 1.25 & 0.92 & 0.23 \\
\hline $\operatorname{dibenz}(\mathrm{a}, \mathrm{h})$ anthracene & n.d. & 10.8 & 0.90 & 1.93 & 0.09 & 0.29 & 0.17 & 0.05 \\
\hline benzo(ghi)perylene & 0.19 & 5.69 & 2.36 & 1.67 & 0.50 & 1.24 & 0.89 & 0.23 \\
\hline anthanthrene & n.d. & 6.94 & 1.67 & 1.77 & 0.08 & 0.30 & 0.18 & 0.06 \\
\hline
\end{tabular}


Table 1. Continued.

\begin{tabular}{|c|c|c|c|c|c|c|c|c|}
\hline \multirow[t]{2}{*}{ Compounds } & \multicolumn{4}{|c|}{ Winter $(n=29)$} & \multicolumn{4}{|c|}{ Summer $(n=20)$} \\
\hline & $\min$ & $\max$ & average & $\mathrm{SD}$ & $\min$ & $\max$ & average & SD \\
\hline coronene & 0.05 & 19.0 & 4.23 & 4.60 & 0.30 & 0.89 & 0.60 & 0.16 \\
\hline dibenzo(a,e)pyrene & n.d. & 1.78 & 0.74 & 0.41 & 0.05 & 0.30 & 0.18 & 0.08 \\
\hline subtotal & 2.23 & 83.4 & 35.7 & 18.7 & 5.50 & 47.3 & 16.5 & 12.3 \\
\hline Total Organics & 380 & 2461 & 1192 & 472 & 650 & 3325 & 1683 & 763 \\
\hline UCM hydrocarbons ${ }^{\mathrm{h}}$ & 56.4 & 1938 & 608 & 508 & 130 & 3281 & 823 & 903 \\
\hline Total Organics + UCM & 436 & 4399 & 1800 & 960 & 780 & 6607 & 2506 & 1645 \\
\hline $\mathrm{OC}\left(\mu \mathrm{g} \mathrm{m}^{-3}\right)$ & 3.20 & 15.6 & 9.12 & 3.71 & 4.15 & 17.6 & 9.69 & 3.65 \\
\hline WSOC $\left(\mu \mathrm{g} \mathrm{m}^{-3}\right)$ & 1.71 & 6.95 & 3.76 & 1.28 & 2.15 & 6.03 & 4.10 & 0.90 \\
\hline
\end{tabular}

\footnotetext{
a SD: standard deviation.

$\mathrm{b}$ n.d.: not detected.

${ }^{c}$ CPI, Carbon Preference Index: $\left(\mathrm{C}_{21}+\mathrm{C}_{23}+\mathrm{C}_{25}+\mathrm{C}_{27}+\mathrm{C}_{29}+\mathrm{C}_{31}+\mathrm{C}_{33}+\mathrm{C}_{35}+\mathrm{C}_{37}+\mathrm{C}_{39}\right) /\left(\mathrm{C}_{22}+\mathrm{C}_{24}+\mathrm{C}_{26}+\mathrm{C}_{28}+\mathrm{C}_{30}+\mathrm{C}_{32}+\mathrm{C}_{34}+\mathrm{C}_{36}+\mathrm{C}_{38}+\mathrm{C}_{40}\right)$ for $n$-alkanes; $\left(\mathrm{C}_{20}+\mathrm{C}_{22}+\mathrm{C}_{24}+\mathrm{C}_{26}+\mathrm{C}_{28}+\mathrm{C}_{30}+\mathrm{C}_{32}+\mathrm{C}_{34}\right) /\left(\mathrm{C}_{21}+\mathrm{C}_{23}+\mathrm{C}_{25}+\mathrm{C}_{27}+\mathrm{C}_{29}+\mathrm{C}_{31}+\mathrm{C}_{33}\right)$ for fatty acids and fatty alcohols.

d Plant wax $n$-alkanes are calculated as the excess odd homologues - adjacent homologues average, and the difference from the total $n$-alkanes is the petroleum-derived amount. Negative values of plant wax $n$-alkanes were taken as zero.

e $\mathrm{C}_{5}$-alkene triols: cis-2-methyl-1,3,4-trihydroxy-1-butene, trans-2-methyl-1,3,4-trihydroxy-1-butene, and 3-methyl-2,3,4-trihydroxy-1butene.

${ }^{f}$ MBTCA: 3-methyl-1,2,3-butanetricarboxylic acid.

g Tris(2,4-di-tert-butyl-phenyl)phosphate (TBPP): due to a lack of the authentic standard, TBPP was quantified using 1,3,5-triphenylbenzene.

${ }^{\mathrm{h}}$ Unresolved Complex Mixture (UCM) of hydrocarbons. UCM estimated on the basis of U/R=5 (unresolved/resolved compounds) for emissions from vehicle traffic in a Los Angeles tunnel (Fraser et al., 1998).
}

a source specific tracer for conifers. Vanillin (VI) and syringic acid (VII) are detectable in pine wood smoke (Simoneit, 2002). Shonanin (VIII) is a predominant lignan of soft wood smoke (e.g., pine). These tracers showed similar temporal trends each other with higher concentrations in winter than in summer (Fig. 4d-i).

Terpenoid biomarkers are present in vegetation smoke, both as natural and thermally altered products (Medeiros and Simoneit, 2008). Dehydroabietic acid (IX) was the dominant diterpenoid in the aerosols, followed by 7-oxodehydroabietic acid (X), indicating a dominant softwood burning. Regular diterpenoid acids such as abietic acid (XI) or pimaric acid (XII) were also detectable (Table 1). $\alpha / \beta$-Amyrins (XIV, $\mathrm{XV}$ ) have been reported in soft wood smokes (Oros and Simoneit, 2001; Simoneit, 2002; Medeiros and Simoneit, 2008), Amazonian smoke aerosol (Bin Abas et al., 1995), and urban aerosols in China (Simoneit et al., 1991). They are triterpenoids that are important constituents of many vascular plants, especially in the gums and mucilages of angiosperms. The concentration ranges of $\alpha+\beta$-amyrin were $0.07-5.96 \mathrm{ng} \mathrm{m}^{-3}$ (average $1.93 \mathrm{ng} \mathrm{m}^{-3}$ ) in winter and 0.34 $3.60 \mathrm{ng} \mathrm{m}^{-3}\left(1.22 \mathrm{ng} \mathrm{m}^{-3}\right)$ in summer with a temporal pattern similar to those of dehydroabietic acid or shonanin (Fig. 4h-j).

In India, biofuel is a major domestic energy source for cooking and heating. Cholesterol (XVI) is a tracer for smoke particles generated from meat cooking and also has been proposed as a source marker of marine organisms ( $\mathrm{Si}$ moneit and Elias, 2000). Stigmasterol (XVII) is a tracer used to identify cow dung smoke (Sheesley et al., 2003). $\beta$ Sitosterol (XVIII), together with stigmasterol, is present in terrestrial higher plants and emitted to the air via biomass burning (Simoneit, 2002; Kawamura et al., 2003). These sterols were more abundant at nighttime than daytime, especially in winter (Fig. 4k-m). Their total concentrations were $1.55-195 \mathrm{ng} \mathrm{m}^{-3}\left(55.0 \mathrm{ng} \mathrm{m}^{-3}\right)$ in winter and $2.14-$ $119 \mathrm{ng} \mathrm{m}^{-3}\left(24.8 \mathrm{ng} \mathrm{m}^{-3}\right)$ in summer (Fig. 3h). The abundance of sterols, lignin products, and terpenoid biomarkers, as well as anhydrosugars, in the troposphere over tropical India indicates that biomass/biofuel burning is an important source of organic aerosols in this region, especially during nighttime in winter.

\subsection{Emissions from plastics}

Plastics are versatile polymeric materials produced and used worldwide (Simoneit et al., 2005). Phthalic acid esters (phthalates) are used as plasticizers in resins and polymers. They can be released into the air from the matrix by evaporation because they are not chemically bonded to the polymer. Attention has been paid to phthalates due to their potential carcinogenic and endocrine disrupting properties (Sidhu et al., 2005). Five phthalates were detected in this study, i.e., dimethyl, diethyl, diisobutyl, di- $n$-butyl, 

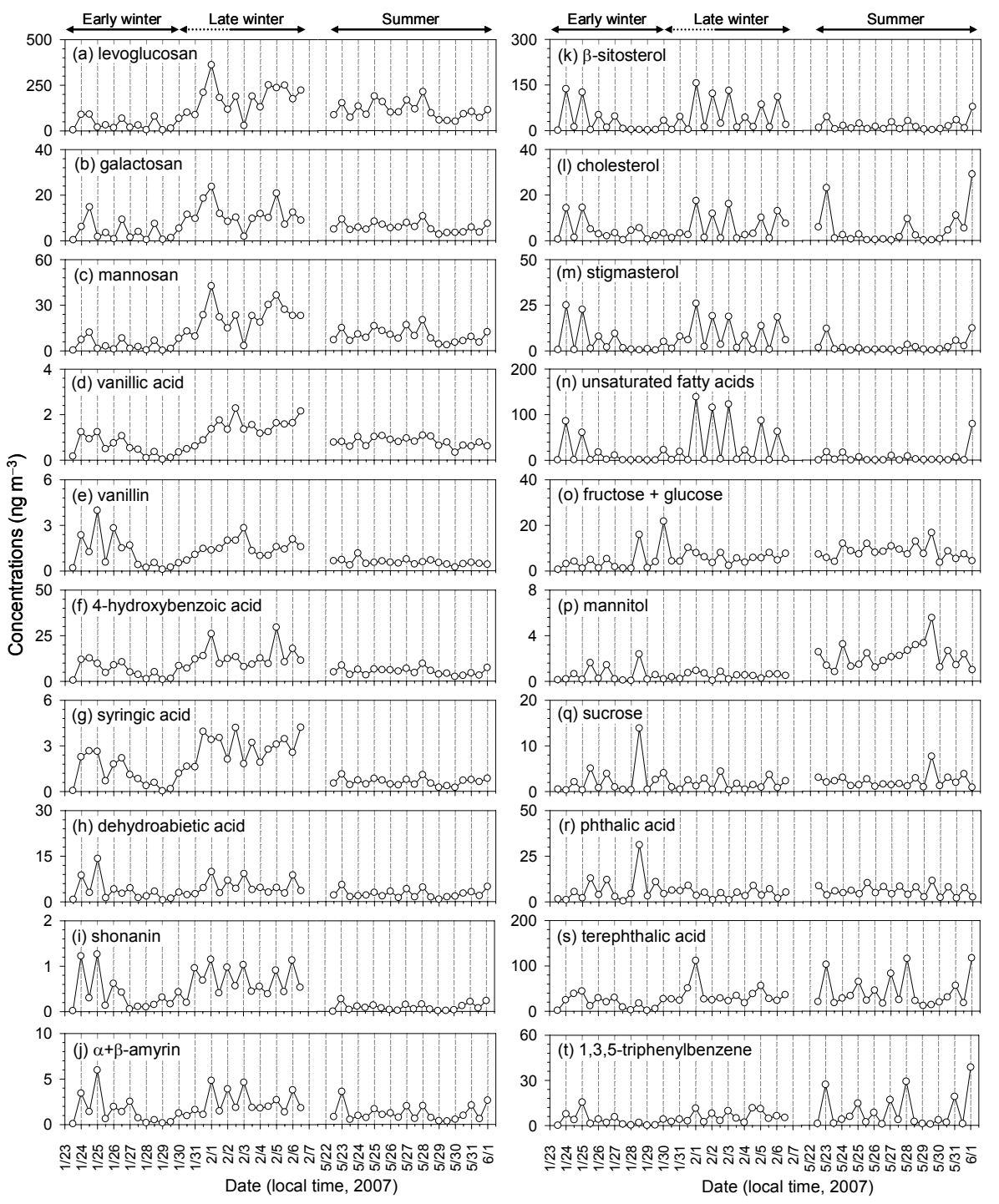

Fig. 4. Temporal variations in the concentrations of biomass-burning tracers and other individual organic compounds detected in the tropical Indian aerosols.

and di-(2-ethylhexyl) (DEHP, see XIX in Fig. A1) phthalates (Fig. 5). The concentrations of phthalates were 295$857 \mathrm{ng} \mathrm{m}^{-3}\left(553 \mathrm{ng} \mathrm{m}^{-3}\right)$ in summer versus $175-598 \mathrm{ng} \mathrm{m}^{-3}$ $\left(303 \mathrm{ng} \mathrm{m}^{-3}\right)$ in winter (Table 1). Higher concentrations observed in summer (Fig. 3j) may be caused by enhanced emission of phthalates from plastics because of the higher ambient temperatures. Similarly, Wang et al. (2006a) also reported that concentrations of phthalates in summer were higher than those in winter. However, the nighttime concentrations of DEHP in Chennai were higher than those at daytime (Fig. 5). This feature is different from other studies and will be discussed below.

In India, most of the municipal solid wastes including a large amount of plastics are generally disposed into open landfills. Plastics are combustible. But under open-fire conditions they can release numerous compounds into the atmosphere (Simoneit et al., 2005). Simoneit et al. (2005) reported that 1,3,5-triphenylbenzene (XXI) can be used as a specific tracer for the open-burning of plastics, especially when coupled with the presence of the antioxidant tris(2,4di-tert-butyl-phenyl)phosphate (TBPP, XX). A good correlation between 1,3,5-triphenylbenzene and TBPP was found (Fig. 6a). In this study, we detected TBPP in most of the samples with higher concentrations at nighttime than at daytime (Fig. 7), indicating an enhanced plastic burning at night. This feature has also been reported in Algiers metropolitan area (Yassaa et al., 2001). Such a refuse burning event may explain the higher concentrations of DEHP at nighttime than at daytime. 


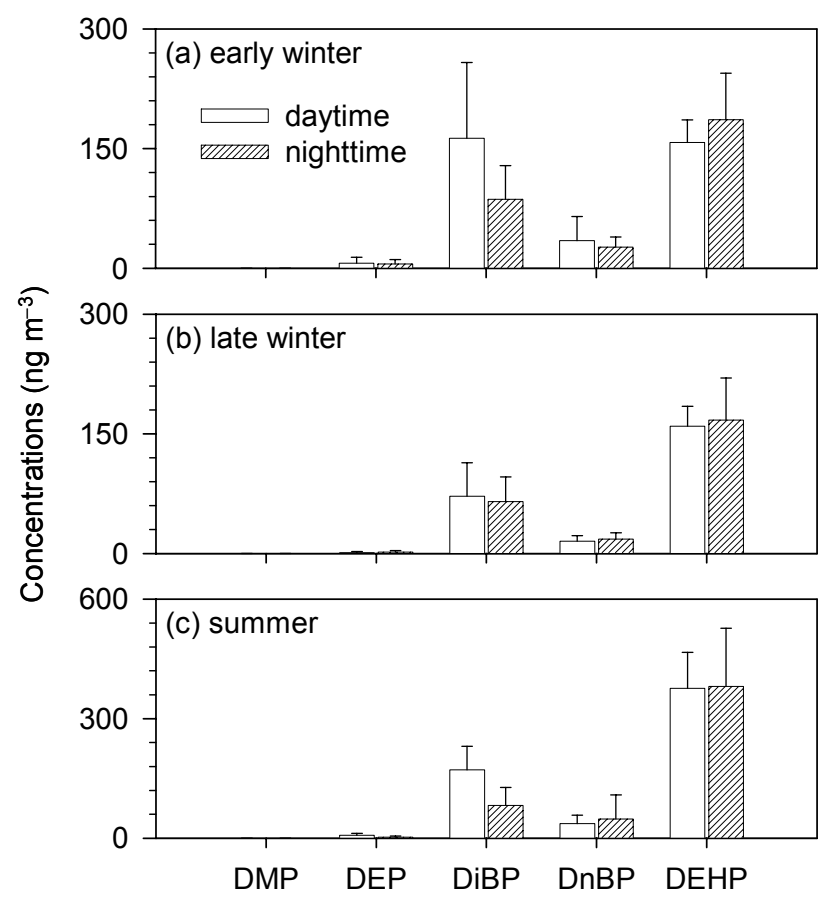

Fig. 5. Molecular distributions of phthalate esters in $\mathrm{PM}_{10}$ aerosols collected in Chennai, tropical India.

\subsection{Fossil fuel combustions}

Hopanes (hopanoid hydrocarbons, XXIII) are specific biomarkers of petroleum and coal (Simoneit et al., 1991; Rogge et al., 1993; Schauer et al., 1999, 2002). They may be emitted into the atmosphere from internal combustion engines and the use of coal. A series of hopanes $\left(\mathrm{C}_{27}-\mathrm{C}_{35}\right.$, but no $\mathrm{C}_{28}$, see Table 1 and Fig. 8) were detected in the Chennai aerosols with the dominance of $\mathrm{C}_{29} \alpha \beta$. Such a molecular distribution has been reported in urban aerosols in China (Simoneit et al., 1991). Their average concentrations were $14.4 \pm 9.09 \mathrm{ng} \mathrm{m}^{-3}$ in winter and $4.97 \pm 1.91 \mathrm{ng} \mathrm{m}^{-3}$ in summer, which are higher than those reported in fourteen Chinese mega-cities $\left(3.1 \pm 4.6 \mathrm{ng} \mathrm{m}^{-3}\right.$ ) (Wang et al., 2006a), Tokyo $\left(0.7-15 \mathrm{ng} \mathrm{m}^{-3}\right.$, average $5.5 \mathrm{ng} \mathrm{m}^{-3}$ ) (Kawamura et al., 1995), Auckland $\left(5.7 \pm 4.3 \mathrm{ng} \mathrm{m}^{-3}\right)$ and Christchurch $\left(2.0 \pm 2.4 \mathrm{ng} \mathrm{m}^{-3}\right)$ in New Zealand (Wang et al., 2006b), suggesting a severe air pollution in India. It should be noted that hopanes are expected to react with $\mathrm{OH}$ radical in the atmosphere, causing a loss of these compounds in ambient particles, especially in summer (Robinson et al., 2006).

Twenty PAHs (3- to 7-ring) were detected in the Chennai aerosols ranging from phenanthrene to dibenzo(a,e)pyrene. Their total concentrations were $35.7 \pm 18.7 \mathrm{ng} \mathrm{m}^{-3}$ in winter versus $16.5 \pm 12.3 \mathrm{ng} \mathrm{m}^{-3}$ in summer, which are similar to those observed in Chinese mega-cities $\left(28 \pm 4.8 \mathrm{ng} \mathrm{m}^{-3}\right.$ in summer) (Wang et al., 2006a). The sources of PAHs include coal and natural gas combustion, automobile emissions, and biomass burning. The diagnostic ratios of IP/(IP+BghiP) were $0.44 \pm 0.04$ at daytime and $0.45 \pm 0.03$ at nighttime, suggesting a mixed source of traffic and coal burning. Among the PAHs detected, benzo(b)fluoranthene $(\mathrm{BbF})$ was the dominant species in winter (Table 1 and Fig. 9). However in summer, 1,3,5-triphenylbenzene became the most abundant, especially at night (Figs. 4t and 9). This suggests that severe plastic burning activities frequently happened during summer nighttime in tropical India as mentioned above.

\subsection{Aromatic acids}

Seven aromatic acids, i.e., benzoic acid, three toluic acids ( $o$ ,$m-$, and $p$-isomers), and three phthalic acids $(o-, m-$, and $p$-isomers), were detected in the samples. They can play an important role to enhance the atmospheric new particle formation (Zhang et al., 2004). The total concentrations of aromatic acids on average were $36.5 \pm 21.9 \mathrm{ng} \mathrm{m}^{-3}$ in winter versus $51.8 \pm 33.9 \mathrm{ng} \mathrm{m}^{-3}$ in summer. Except for terephthalic acid (Fig. 4s), all the aromatic acids showed higher concentrations at daytime than at nighttime (Fig. 10), indicating a photochemical source.

Benzoic acid is proposed to be a primary pollutant in the exhaust of motor vehicles (Kawamura and Kaplan, 1987; Rogge et al., 1993; Kawamura et al., 2000) and a secondary product from photochemical degradation of aromatic hydrocarbons emitted by automobiles (Suh et al., 2003). The concentration ranges of benzoic acid were $0.54-2.16 \mathrm{ng} \mathrm{m}^{-3}$ (average $1.25 \mathrm{ng} \mathrm{m}^{-3}$ ) in winter and 0.95 $2.56 \mathrm{ng} \mathrm{m}^{-3}\left(1.44 \mathrm{ng} \mathrm{m}^{-3}\right)$ in summer (Table 1). The concentration ranges of total toluic acids were $0.15-1.18 \mathrm{ng} \mathrm{m}^{-3}$ $\left(0.62 \mathrm{ng} \mathrm{m}^{-3}\right)$ in winter and $0.33-1.09 \mathrm{ng} \mathrm{m}^{-3}\left(0.59 \mathrm{ng} \mathrm{m}^{-3}\right)$ in summer. They may be derived from the oxidation of xylene (Forstner et al., 1997) or directly emitted from motor vehicle exhausts (Kawamura et al., 2000). The relatively low concentrations of benzoic and toluic acids in the tropical Indian aerosols may be explained by their presence mainly in gas phase (Kawamura et al., 2000; Fraser et al., 2003). The molecular distribution of phthalic acids was characterized by the predominance of terephthalic acid (Fig. 10). This pattern is different from those reported in aerosols from other studies (Wang et al., 2006a; Fu et al., 2008). Interestingly, a good correlation was found between 1,3,5-triphenylbenzene and terephthalic acid, whereas no correlation was obtained between 1,3,5-triphenylbenzene and phthalic acid in this study (Fig. 6b). This suggests that the open burning of plastics as well can produce terephthalic acid. Terephthalic acid may in part be produced by the hydrolysis of tere-phthalate during the open burning of municipal refuses including plastics.

\subsection{Aliphatic lipids}

Homologous $n$-alkanes $\left(\mathrm{C}_{18}-\mathrm{C}_{40}\right)$ in the aerosol samples were detected with higher concentrations at nighttime (Fig. 11). Their concentrations were $30.9-727 \mathrm{ng} \mathrm{m}^{-3}$ 

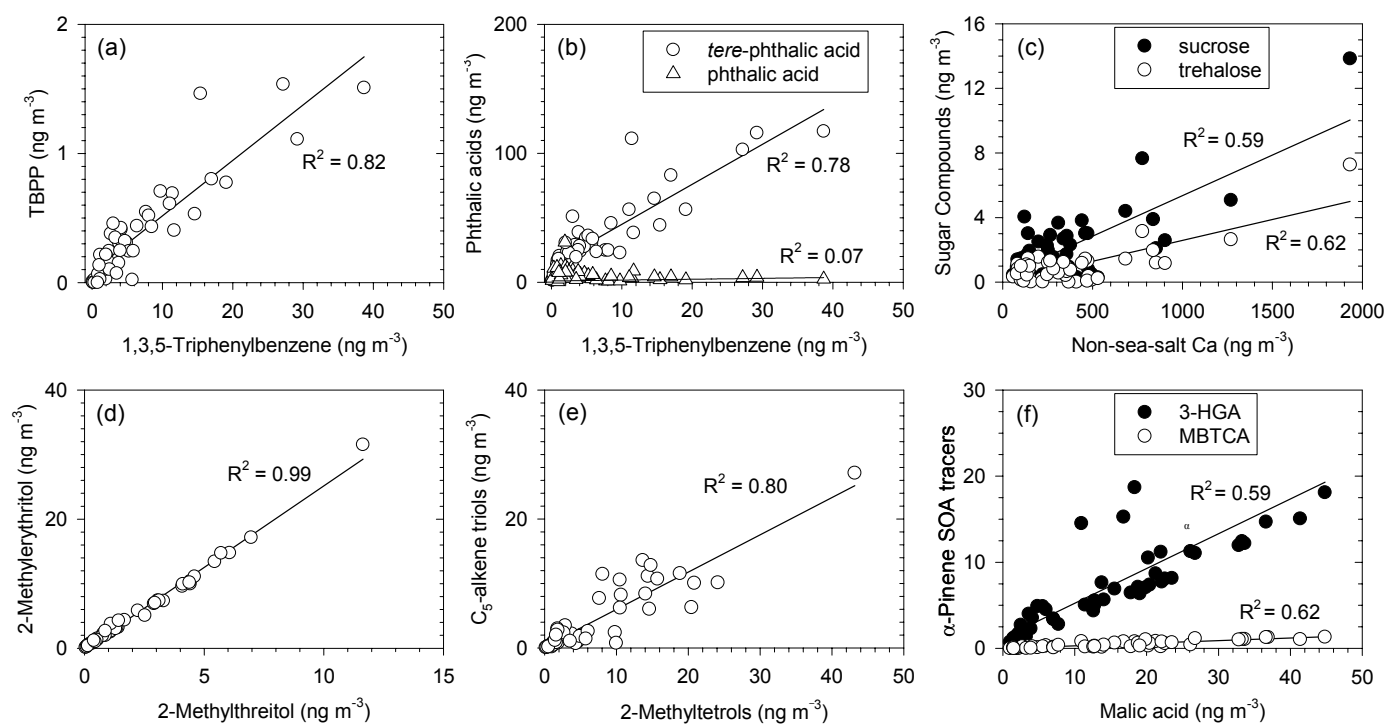

Fig. 6. Correlations between the concentrations of (a) 1,3,5-triphenylbenzene (a tracer for plastic burning) and tris(2,4-di-tert-butylphenyl)phosphate (TBPP), (b) 1,3,5-triphenylbenzene and phthalic acids, (c) non-sea-salt Ca and two sugar compounds (sucrose and trehalose), (d) 2-methylthreitol and 2-methylerythritol, (e) 2-methyltetrols and $\mathrm{C}_{5}$-alkene triols, and (f) malic acid and two $\alpha$-pinene SOA tracers (3-HGA and MBTCA).

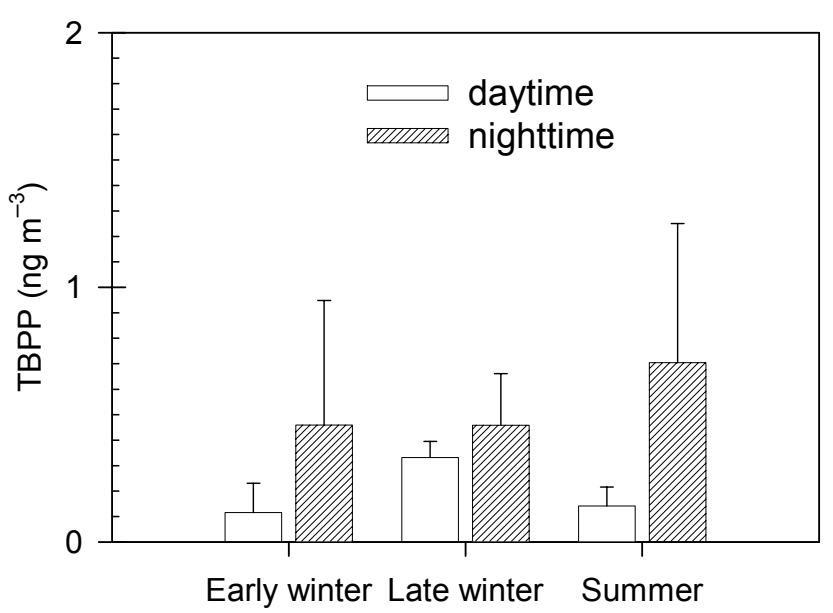

Fig. 7. Concentrations of tris(2,4-di-tert-butylphenyl)phosphate (TBPP) in $\mathrm{PM}_{10}$ aerosols collected in Chennai, tropical India.

(average $187 \mathrm{ng} \mathrm{m}^{-3}$ ) in summer and $15.2-437 \mathrm{ng} \mathrm{m}^{-3}$ $\left(141 \mathrm{ng} \mathrm{m}^{-3}\right)$ in winter. Their molecular distributions are characterized by weak odd-carbon-numbered predominance with a maximum at $\mathrm{C}_{29}$ (CPI ranged from 1.17-2.34). Such a molecular distribution suggests that they are mainly derived from the incomplete combustion of fossil fuels, especially for lower molecular weight $n$-alkanes such as $\mathrm{C}_{20}-\mathrm{C}_{26}$. However, higher molecular weight $n$-alkanes are likely derived from higher plant waxes, in which $\mathrm{C}_{27}, \mathrm{C}_{29}, \mathrm{C}_{31}$ and $\mathrm{C}_{33}$ are dominant species (Gagosian et al., 1982). Interestingly
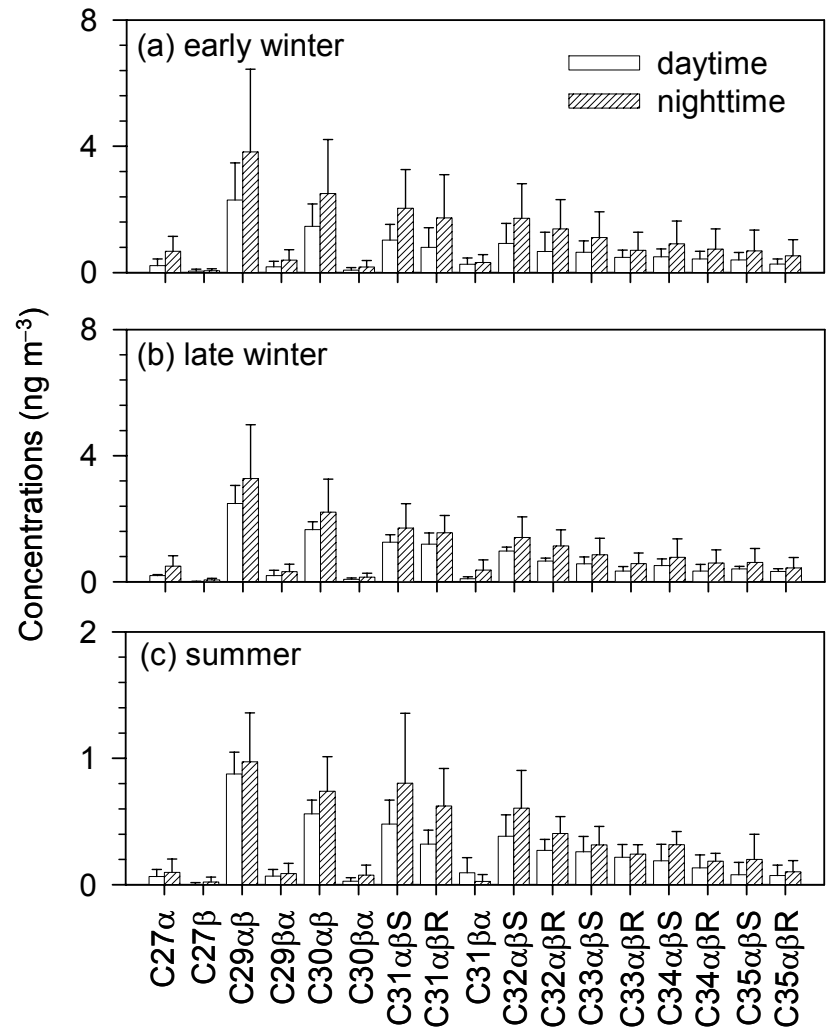

Fig. 8. Molecular distributions of hopanes in $\mathrm{PM}_{10}$ aerosols collected in Chennai, tropical India. 


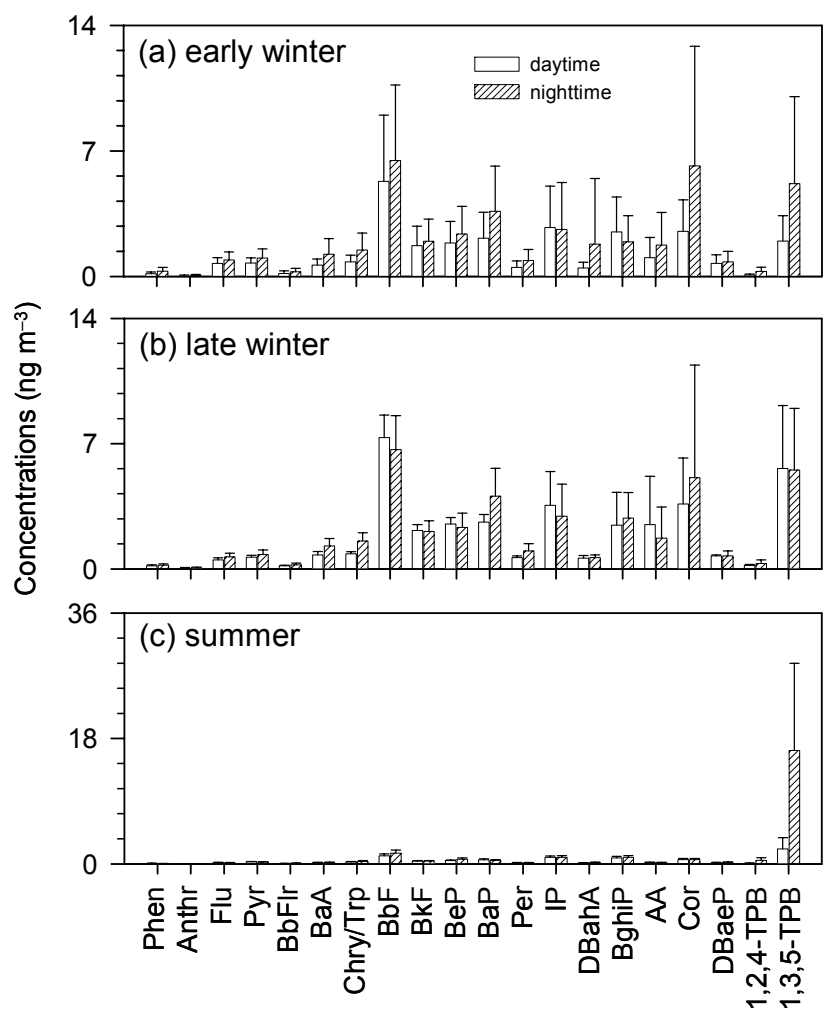

Fig. 9. Molecular distributions of PAHs in $\mathrm{PM}_{10}$ aerosols collected in Chennai, tropical India.

has been successfully used for a series of, carbon numbers of detected $n$-alkane were up to $\mathrm{C}_{40}$ (Fig. 11). Such a feature has been reported in smokes from landfill plastic burning test (Simoneit et al., 2005). These long-chain alkanes $\left(>\mathrm{C}_{37}\right)$ can also be interpreted by the high-molecular-weight paraffin wax, a petroleum product used to coat the inner surface of food containers (e.g., paper cartons for milk and juice) (Simoneit et al., 2004). These findings again suggest that the open-burning of municipal wastes is an important source of organic aerosols in tropical India.

Plant wax $n$-alkanes are attributable to vascular plant waxes. They are calculated as the excess of odd homologues minus adjacent even homologues (Simoneit et al., 2004) and shown in Table 1. In summer, the concentration range of total plant wax $n$-alkanes was $4.85-76.3 \mathrm{ng} \mathrm{m}^{-3}\left(22.8 \mathrm{ng} \mathrm{m}^{-3}\right)$, which are comparable to those in winter $\left(2.26-49.6 \mathrm{ng} \mathrm{m}^{-3}\right.$, $19.8 \mathrm{ng} \mathrm{m}^{-3}$ ), suggesting that there is no significant difference in higher plant emissions between cold and warm seasons in tropical India. This feature may be associated with the vegetation types and relatively high ambient temperature even in winter $\left(14.2-34.9^{\circ} \mathrm{C}\right.$, average $\left.23^{\circ} \mathrm{C}\right)$ during the campaign.

A homologous series of straight chain fatty acids, including $\mathrm{C}_{8: 0}-\mathrm{C}_{34: 0}$ saturated and unsaturated acids (e.g., $\mathrm{C}_{16: 1}$ and $\mathrm{C}_{18: 1}$ ), were detected in the aerosols with higher nighttime concentrations (Figs. $3 \mathrm{~b}$ and $4 \mathrm{n}$ ). Concentration ranges

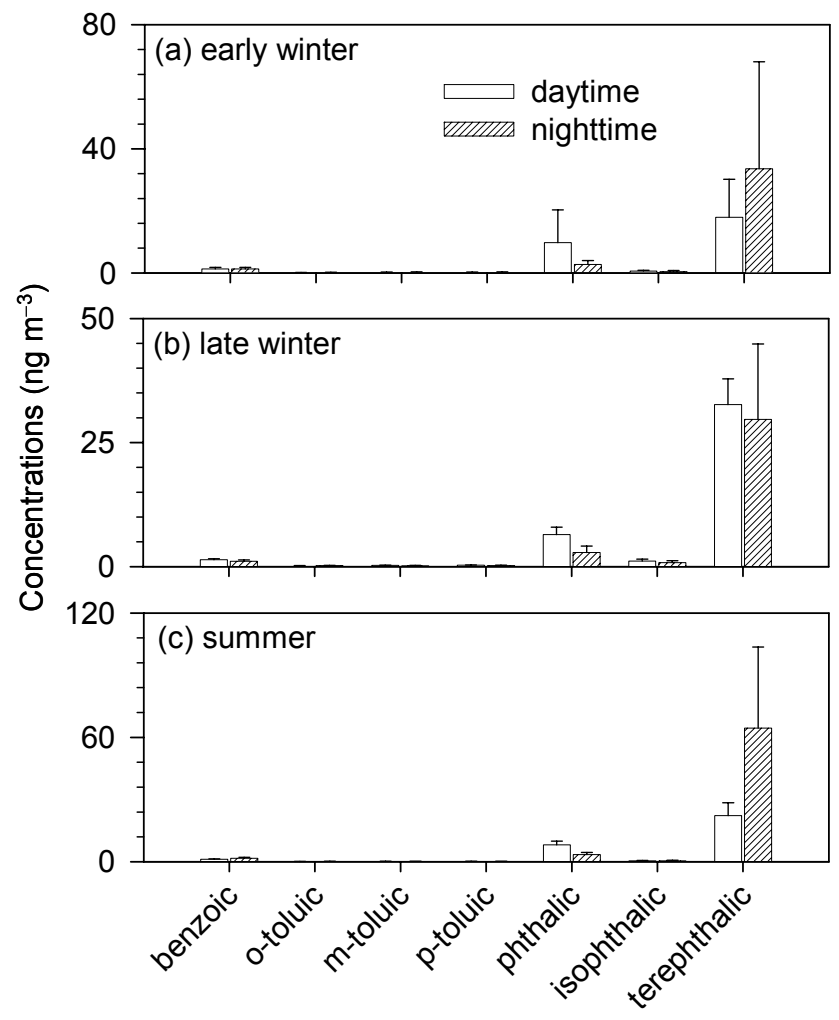

Fig. 10. Molecular distributions of aromatic acids in $\mathrm{PM}_{10}$ aerosols collected in Chennai, tropical India.

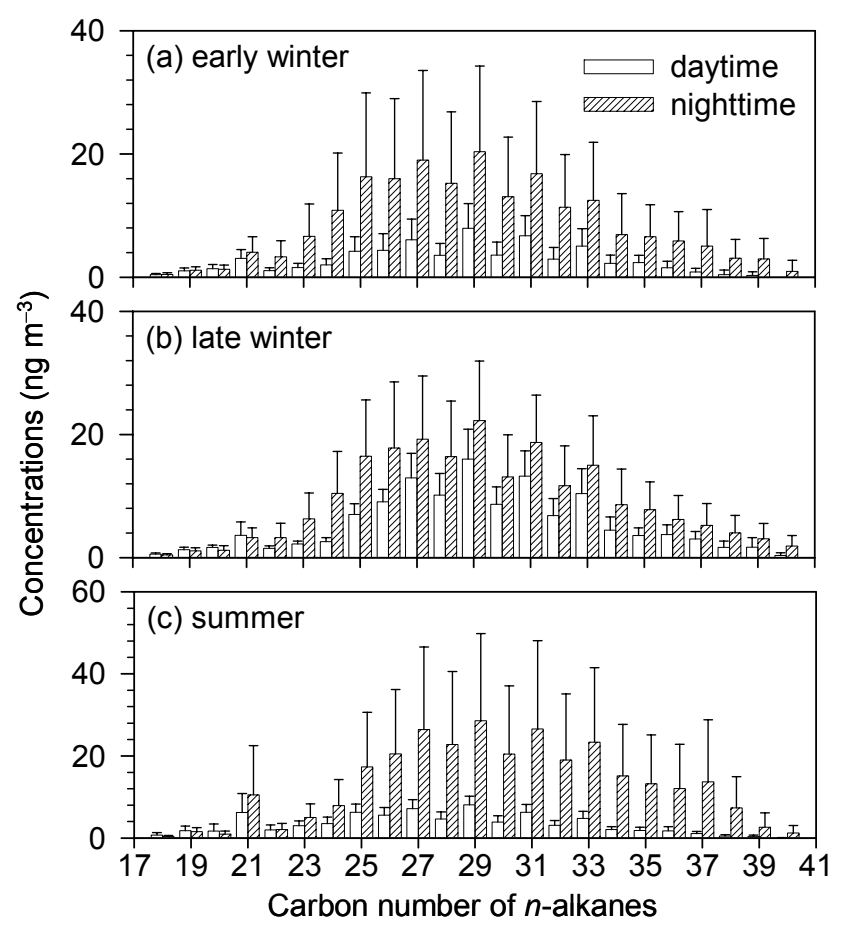

Fig. 11. Molecular distributions of $n$-alkanes in $\mathrm{PM}_{10}$ aerosols collected in Chennai, tropical India. 
were $40.5-657 \mathrm{ng} \mathrm{m}^{-3}\left(302 \mathrm{ng} \mathrm{m}^{-3}\right)$ in winter and $141-$ $1448 \mathrm{ng} \mathrm{m}^{-3}\left(504 \mathrm{ng} \mathrm{m}^{-3}\right)$ in summer, which are higher than those of $n$-alkanes and fatty alcohols. Their molecular distributions are characterized by a strong even carbon number predominance with two maxima at $\mathrm{C}_{16: 0}$ and $\mathrm{C}_{24: 0}$ (CPI ranged from 2.80-6.40, Table 1 and Fig. 12). A similar bimodal distribution has been reported in continental and marine aerosols (Mochida et al., 2002; Kawamura et al., 2003; Fu et al., 2008).

The concentration ratios of lower molecular weight fatty acids (LFAs, $<\mathrm{C}_{20: 0}$ ) to higher molecular weight fatty acids (HFAs, $\mathrm{C}_{20: 0}-\mathrm{C}_{34: 0}$ ) were $5.3 \pm 1.8$ in summer versus $1.4 \pm 0.8$ in winter. These values are higher than those reported in Mt. Tai aerosols (average ratio of LFAs/HFAs was $1.02 \pm 0.80$ ) in Central East China (Fu et al., 2008). HFAs are derived from terrestrial higher plant wax whereas LFAs have multiple sources such as vascular plants, microbes, marine phytoplankton, and kitchen emissions (Rogge et al., 1991; Schauer et al., 2001). Thus, our results suggest that much more LFAs are emitted from microbial sources in tropical India due to higher temperature and humidity, especially in summer.

The presence of unsaturated fatty acids in aerosols is indicative of recent biogenic inputs from higher plants as well as microbial/marine sources. In urban environments, cooking, motor vehicles, and biomass burning can also be the major anthropogenic sources of these acids (Rogge et al., 1993, 1996). They can be rapidly oxidized once emitted to the atmosphere (Kawamura and Gagosian, 1987). Oleic $\left(\mathrm{C}_{18: 1}\right)$ and linoleic $\left(\mathrm{C}_{18: 2}\right)$ acids were detected as dominant species in the Chennai aerosols. Their total concentrations showed a clear diurnal trend with a peak at nighttime (Fig. 4n), suggesting an enhanced emission to the atmosphere. Moreover, significantly high concentrations of unsaturated fatty acids were observed at nighttime during late winter when the air mass mainly originated from Southeast Asia over the Bay of Bengal, indicating that marine air masses enriched with unsaturated fatty acids should be transported to Chennai.

Oleic acid is also a good proxy for unsaturated organic matter in atmospheric aerosols and a good model compound for studying aerosol reactivity (Rudich et al., 2007). The ratios of oleic acid to stearic acid $\left(\mathrm{C}_{18: 1} / \mathrm{C}_{18: 0}\right)$ in winter ranged from $0.01-0.18$ (average 0.05 ) and 0.04-1.05 (0.61) during daytime and nighttime, respectively. On the other hand, the ratios of $\mathrm{C}_{18: 1} / \mathrm{C}_{18: 0}$ were lower in summer ranging from $0-0.05(0.01)$ at daytime and 0.04-0.35 (0.10) at nighttime. This again suggests an enhanced photochemical degradation of oleic acid occurs during daytime, especially in summer. Wang et al. (2006a) reported that the ratios of unsaturated fatty acids $\left(\mathrm{C}_{16: 1}+\mathrm{C}_{18: 1}\right)$ to saturated fatty acids $\left(\mathrm{C}_{16: 0}+\mathrm{C}_{18: 0}\right)$ in urban aerosols from fourteen Chinese megacities were $1.14 \pm 0.98$ in winter versus $0.43 \pm 0.09$ in summer. In the Chennai aerosols, the values $(0.12 \pm 0.15$ in winter versus $0.01 \pm 0.02$ in summer) are about one order of magnitude lower than those reported in Chinese megacities,

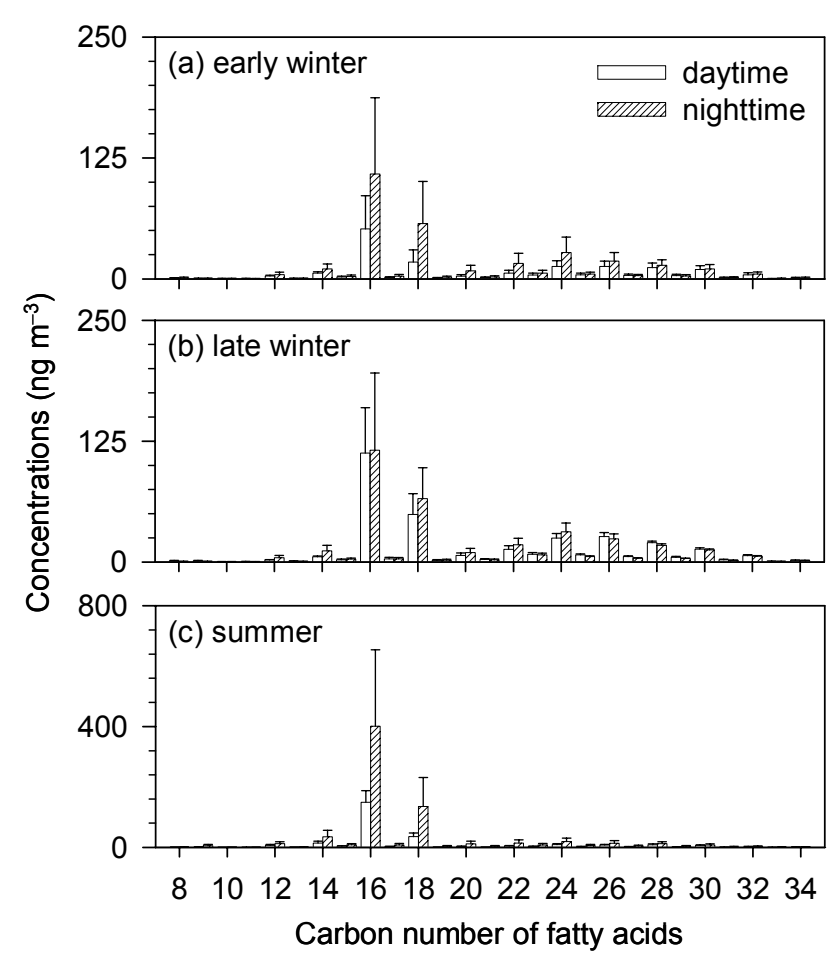

Fig. 12. Molecular distributions of fatty acids in $\mathrm{PM}_{10}$ aerosols collected in Chennai, tropical India.

indicating that the photooxidation of unsaturated fatty acids in tropical region is more significant under stronger radiation conditions.

Normal $\mathrm{C}_{14}-\mathrm{C}_{34}$ fatty alcohols were detected in the aerosols. Their distributions are characterized with strong even carbon number predominance (CPIs are 9.75 \pm 2.94 in winter and $10.9 \pm 6.77$ in summer) with $C_{\max }$ at $C_{28}$ or $C_{30}$ (Fig. 13). Total concentrations of $n$-alcohols were 11.0 $116 \mathrm{ng} \mathrm{m}^{-3}\left(67.9 \mathrm{ng} \mathrm{m}^{-3}\right)$ in winter and $21.0-155 \mathrm{ng} \mathrm{m}^{-3}$ $\left(70.4 \mathrm{ng} \mathrm{m}^{-3}\right.$ ) in summer (Fig. 3c). Long-chain fatty alcohols are abundant in higher plant waxes, soils, and loess deposits, whereas the homologues of $<\mathrm{C}_{20}$ are abundant in soil microbes and marine biota (Simoneit et al., 1991). It should be noted that biomass burning can also produce a large amount of fatty alcohols, together with $n$-alkanes and fatty acids (Simoneit, 2002). The CPI values together with the molecular distributions of fatty alcohols suggest that they are mainly derived from higher plant waxes and partly from microbial and/or marine emissions both in winter and in summer.

\subsection{Sugars/sugar alcohols}

Eleven sugar compounds (glycerol, erythritol, xylose, arabitol, fructose, glucose, mannitol, inositol, sucrose, trehalose, and maltose) were detected in the tropical Indian aerosols (Fig. 14). These compounds are completely watersoluble and thus contribute to WSOC in aerosol. They have 


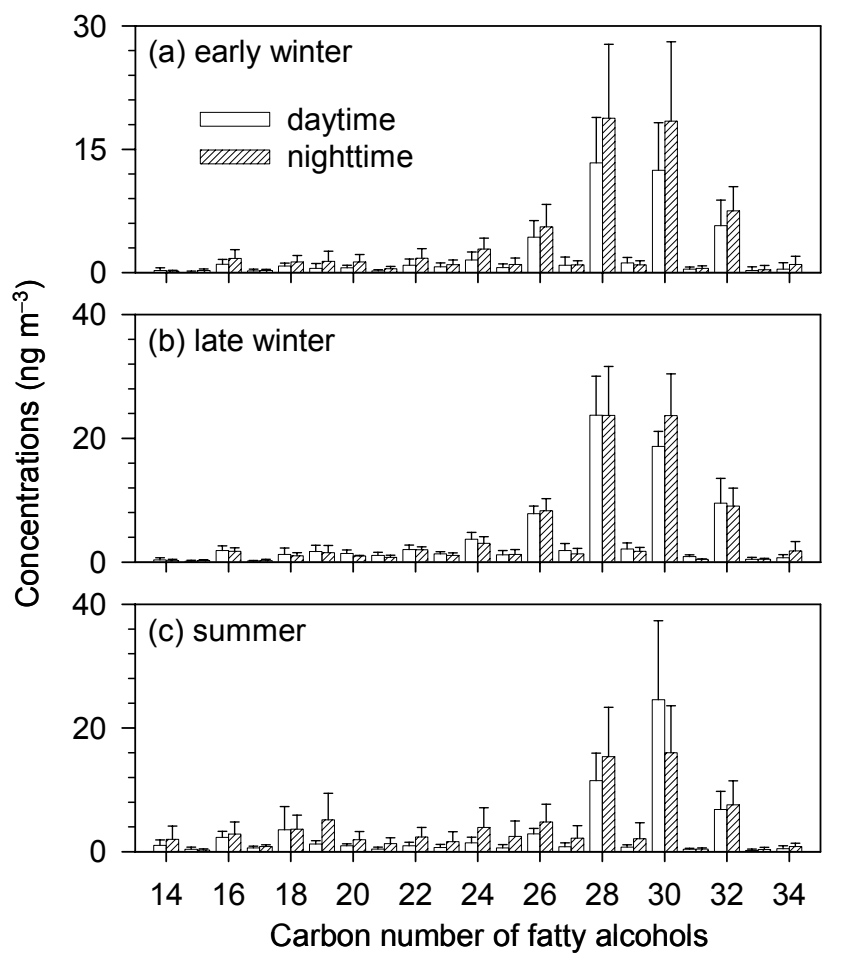

Fig. 13. Molecular distributions of fatty alcohols in $\mathrm{PM}_{10}$ aerosols collected in Chennai, tropical India.

been proposed as tracers for resuspension of surface soil and unpaved road dust, which contain biological materials including pollen, fungi and bacteria (Graham et al., 2003; Simoneit et al., 2004; Yttri et al., 2007). Concentrations of total sugars/sugar alcohols were more abundant in summer $\left(29.5 \pm 7.83 \mathrm{ng} \mathrm{m}^{-3}\right)$ than in winter $\left(20.0 \pm 11.8 \mathrm{ng} \mathrm{m}^{-3}\right)(\mathrm{Ta}-$ ble 1), suggesting higher biological activities in summer.

Most of them were found to be more abundant at daytime than nighttime (Figs. 3e, 4o-q and 14). Fructose and glucose, together with sucrose (XXV), have been proposed to be released as pollen, fern spores, and other "giant" bioaerosol particles during daytime (Graham et al., 2003). The direct emission from developing leaves is also an important source of sugars and sugar alcohols. It should be noted that sugar compounds can further be emitted through biomass burning. For example, maltose and sucrose have been reported in wood smoke (Nolte et al., 2001). Medeiros and Simoneit (2008) reported that a large amount of sugars (e.g., maltose) and sugar alcohols (e.g., erythritol - XXIV, arabitol, and mannitol) can be emitted upon combustion of green vegetation from temperate forests.

In order to get further insight to the sources of sugars in the tropical Indian aerosols, Principal Component Analysis (PCA) was performed using the concentrations of various sugars (including anhydrosugars) as variables. PCA is a useful approach for verifying the sources of aerosols (Hopke, 1985) and has been successfully used for a series

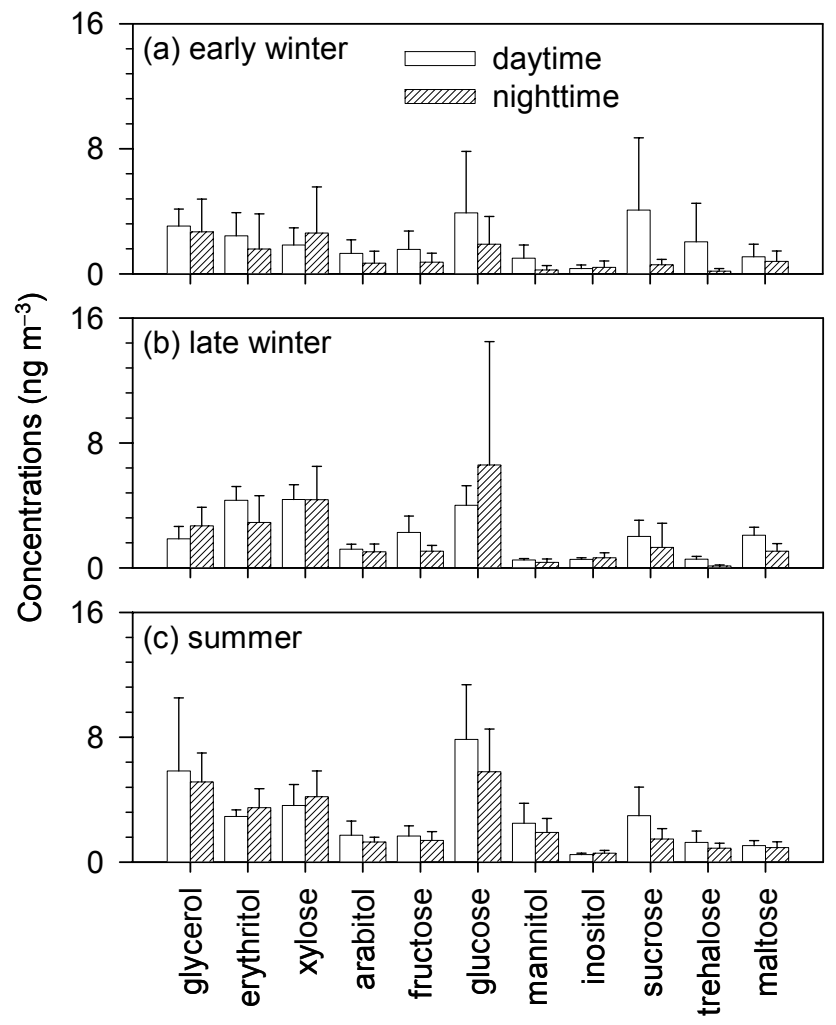

Fig. 14. Molecular distributions of sugar compounds in $\mathrm{PM}_{10}$ aerosols collected in Chennai, tropical India.

of Dicarboxylic Acids (DCAs) and other atmospheric trace species (Wolff and Korsog, 1985; Kawamura and Sakaguchi, 1999; Mochida et al., 2003; Wan and Yu, 2007; Fu et al., 2008). The datasets of sugars for winter $(n=29)$ and summer $(n=20)$ aerosol samples were subjected to PCA analysis based on their correlation matrix, followed by the varimax rotation of the eigenvectors. Principal component loadings, which are correlation coefficients between the concentrations of individual saccharides with principal components, are shown in Table 2. Three components were set for both winter and summer samples by the scree tests.

For the winter dataset, three components were found to account for $87.2 \%$ of the total variance, with the first component corresponding to $48.1 \%$. Levoglucosan, galactosan, mannosan, erythritol, xylose, inositol, and maltose showed loadings of $>0.87$ in component 1 , which is mainly associated with the emissions from biomass burning. In contrast, mannitol, sucrose, and trehalose showed loadings of $>0.91$ in component $2(29.1 \%)$. Arabitol, fructose, and glucose also showed major loadings of $>0.56$ in component 2 , suggesting a common biological origin. These sugar polyols are abundantly produced by many fungi. They are also the major soluble carbohydrates in the bark of trees, branches and leaves. Trehalose (glucose + glucose) is present in a large variety of microorganisms (fungi, bacteria and yeast), and a few higher 
Table 2. Results of principal component analysis with Varimax rotation for the dataset of sugar compounds (including anhydrosugars) in the urban aerosols from Chennai, tropical India.

\begin{tabular}{|c|c|c|c|c|c|c|}
\hline \multirow{2}{*}{ Sugars } & \multicolumn{3}{|c|}{ Winter } & \multicolumn{3}{|c|}{ Summer } \\
\hline & Component 1 & Component 2 & Component 3 & Component 1 & Component 2 & Component 3 \\
\hline galactosan & 0.87 & 0.10 & 0.34 & 0.92 & -0.23 & -0.02 \\
\hline mannosan & 0.98 & 0.02 & 0.12 & 0.94 & -0.23 & -0.13 \\
\hline levoglucosan & 0.98 & 0.07 & 0.07 & 0.98 & -0.11 & -0.11 \\
\hline glycerol & 0.29 & 0.21 & 0.89 & -0.06 & -0.07 & 0.78 \\
\hline erythritol & 0.92 & 0.33 & 0.11 & 0.91 & 0.02 & 0.25 \\
\hline xylose & 0.93 & -0.02 & 0.28 & 0.93 & -0.03 & -0.20 \\
\hline arabitol & 0.62 & 0.65 & 0.31 & 0.01 & 0.97 & 0.07 \\
\hline fructose & 0.55 & 0.63 & -0.12 & 0.19 & 0.30 & 0.84 \\
\hline glucose & 0.20 & 0.56 & 0 & 0.10 & 0.86 & -0.36 \\
\hline mannitol & 0.12 & 0.91 & 0.22 & -0.09 & 0.92 & -0.01 \\
\hline inositol & 0.87 & 0.11 & 0.37 & 0.82 & 0.29 & 0.14 \\
\hline sucrose & -0.03 & 0.98 & 0.02 & -0.21 & 0.88 & 0.30 \\
\hline trehalose & -0.12 & 0.96 & 0.12 & -0.01 & 0.92 & 0.25 \\
\hline maltose & 0.88 & 0.20 & -0.28 & 0.74 & 0.08 & 0.23 \\
\hline Variance $(\%)$ & 48.1 & 29.1 & 10.0 & 40.8 & 31.8 & 12.9 \\
\hline
\end{tabular}

plants and invertebrates (Medeiros et al., 2006). Sucrose is the predominant sugar present in the phloem of plants and is important in developing flower buds (Bieleski, 1995). They are used to trace the resuspension of surface soil and unpaved road dust (Simoneit et al., 2004). Arabitol and fructose also show loadings of 0.62 and 0.55 in component 1 , respectively. This suggests that during winter these compounds are emitted by biomass burning as well, as mentioned earlier in this section. Glycerol showed a loading of $>0.89$ in component 3 $(10.0 \%)$. It is a reduced sugar primarily produced by fungal metabolism in soils and is resuspended into the atmosphere by wind motion (Simoneit et al., 2004). However, the differences of the estimated sources between components 2 and 3 can not be elucidated at this moment.

In summer, three components were found to account for $85.5 \%$ of the total variance, with the first component corresponding to $40.8 \%$. Levoglucosan, galactosan, mannosan, erythritol, and xylose showed loadings of $>0.91$ in component 1 that is associated with biomass burning. Inositol and maltose also showed loadings of $>0.74$ in component 1 , suggesting a significant contribution from biomass burning. Arabitol, glucose, mannitol, sucrose, and trehalose showed loadings of $>0.86$ in component 2 (31.8\%). As mentioned above, these primary saccharides and sugar polyols could be derived from biological sources or suspended soil dust. Interestingly, positive correlations were obtained between the concentrations of non-sea-salt $\mathrm{Ca}$ (nss-Ca), a tracer for soil dust, and sucrose $\left(R^{2}=0.59\right)$ and trehalose $\left(R^{2}=0.62\right)$ in the Chennai aerosols (Fig. 6c). Bauer et al. (2008) proposed arabitol and mannitol as tracers for the quantification of airborne fungal spores. Graham et al. (2003) reported that glucose and fructose, together with sucrose, showed higher daytime concentrations, which were explained by the specific daytime release of pollen, fern spores and other bioaerosols in summer. Glycerol and fructose showed loadings of 0.78 and 0.84 in component $3(12.9 \%)$, respectively, suggesting that these two compounds are derived from similar sources in tropical India in summer.

\subsection{Hydroxy-/polyacids}

Seven hydroxy- or poly-acids were detected in the tropical Indian aerosols, including glycolic, salicylic, glyceric, malic, tartaric, citric, and tricarballylic acids. Glycolic acid (hydroxyacetic acid), the smallest $\alpha$-hydroxy acid, is the most abundant species in this group during winter. It is hygroscopic and highly water-soluble. However in summer, malic acid is the most abundant hydroxyacid (Table 1). Salicylic acid (2-hydroxybenzoic acid) is highly CCN active (Hartz et al., 2006), although its solubility is low. Little is known about salicylic acid in the atmosphere. Van Pinxteren and Herrmann (2007) detected salicylic acid $\left(0.1 \mathrm{ng} \mathrm{m}^{-3}\right)$ in one $\mathrm{PM}_{10}$ sample collected in a rural site in Germany. Fu et al. (2009b) reported that the concentration range of salicylic acid was $0.002-0.102 \mathrm{ng} \mathrm{m}^{-3}$ (average $0.042 \mathrm{ng} \mathrm{m}^{-3}$ ) in the Arctic aerosols. In the present study, the average concentrations of salicylic acid were $0.38 \pm 0.22 \mathrm{ng} \mathrm{m}^{-3}$ in winter versus $0.27 \pm 0.07 \mathrm{ng} \mathrm{m}^{-3}$ in summer.

Glyceric, malic, tartaric and citric acids are suggested as secondary oxidation products. These compounds are all characterized by a diurnal pattern with higher concentrations during daytime than nighttime (Fig. 15c-f). This trend is opposite to most organic species (such as lipid class compounds) as mentioned in Sect. 3.3, whose diurnal 

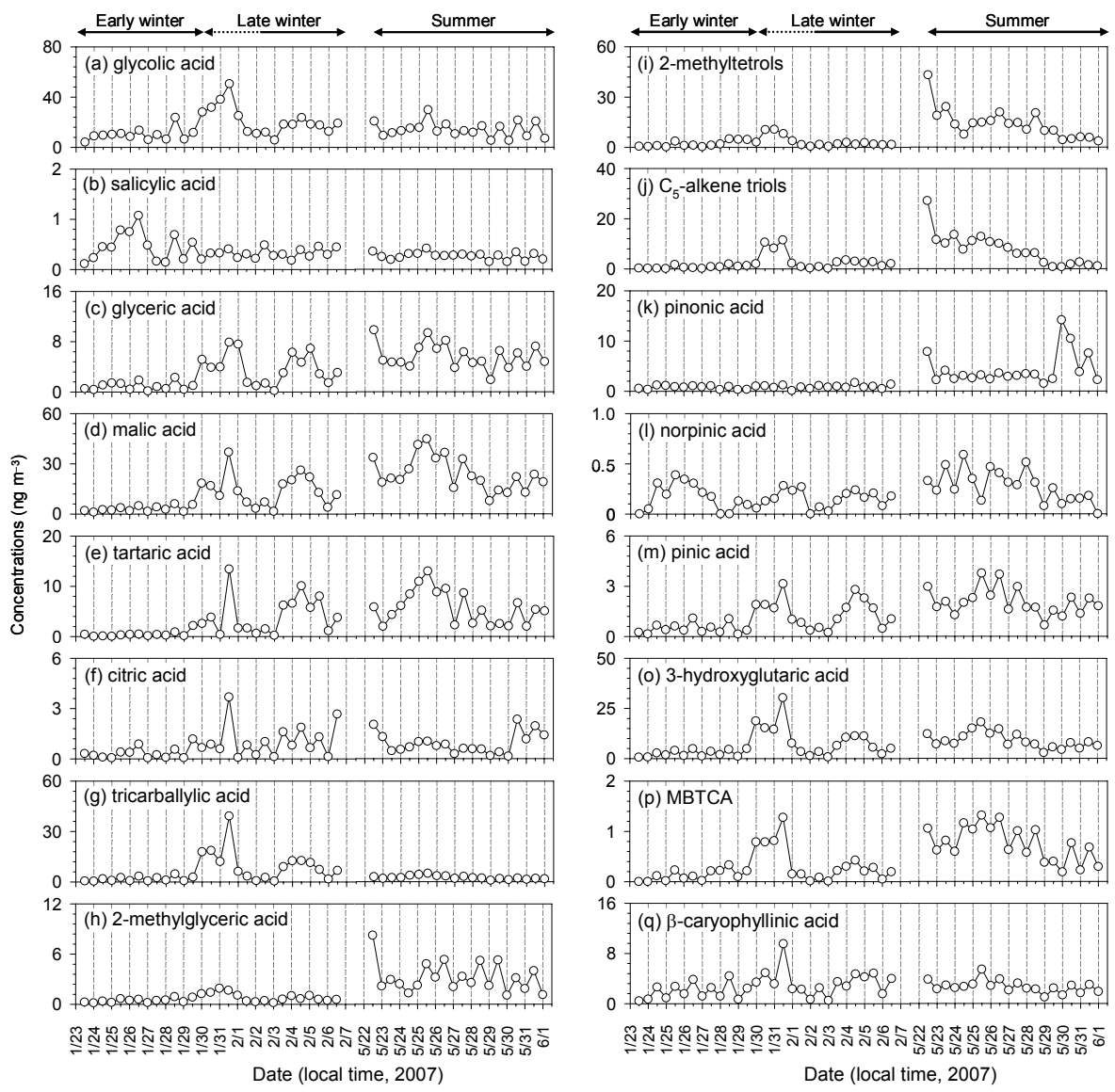

Fig. 15. Temporal variations in the concentrations of hydroxy-/polyacids and biogenic secondary organic aerosol tracers detected in the tropical Indian aerosols.

distributions are controlled by the land/sea breeze patterns in Chennai. In addition, the diurnal patterns of glycolic, salicylic and tricarballylic acids were also characterized by higher concentrations during daytime (Fig. 15), indicating that they are mainly produced by photochemical oxidation. However, the temporal variations of these acids were different from polyacids such as malic acid. Malic acid can be produced by the photochemical oxidation of succinic acid (Kawamura and Ikushima, 1993), which is one of the oxidation products of biogenic unsaturated fatty acids in the atmosphere (Matsunaga et al., 1999). One possible source for salicylic acid is benzoic acid that may react with hydroxyl radicals (Nakajima et al., 2008).

\subsection{Biogenic SOA tracers}

Biogenic VOCs that are released from vegetation include isoprene, monoterpenes, sesquiterpenes, and oxygenated hydrocarbons such as alcohols, aldehydes and ketones (Guenther et al., 2006; Goldstein and Galbally, 2007; Duhl et al., 2008). Considerable efforts have been devoted in the last decade to understand secondary organic aerosol formation from the photooxidation of biogenic VOCs (Hoffmann et al., 1997; Yu et al., 1999; Claeys et al., 2004a; Hallquist et al., 2009), because SOA is an important component in the Earth's atmosphere to influence the atmospheric radiation budget directly by scattering sunlight and indirectly by acting as Cloud Condensation Nuclei (CCN) (Kanakidou et al., 2005). On a global scale, the contribution of biogenic VOCs to SOA $(18.5-270 \mathrm{Tg} / \mathrm{yr})$ is one order of magnitude greater than that of anthropogenic VOCs (1-30 Tg/yr) (Andreae and Crutzen, 1997).

Isoprene (2-methyl-1,3-butadiene, $\mathrm{C}_{5} \mathrm{H}_{8}$ ) is highly reactive because of the presence of $\mathrm{C}=\mathrm{C}$ bonds, which makes it susceptible to react with oxidants (e.g., $\mathrm{OH}, \mathrm{O}_{3}$, and $\mathrm{NO}_{3}$ ). The global emissions of biogenic terpenes and anthropogenic hydrocarbons are both far lower than that of isoprene (500-750 $\mathrm{Tg} \mathrm{yr}^{-1}$ ) (Guenther et al., 2006). Isoprene oxidation products can partition in the gas phase and particles and lead to SOA formation (Claeys et al., 2004a; Hallquist et al., 2009). Six organic compounds were identified as isoprene SOA tracers in the Chennai aerosols, including 2-methylglyceric acid (XXVI), three $\mathrm{C}_{5}$-alkene triols (XXVII-XXIX), and two 2-methyltetrols 
(2-methylthreitol and 2-methylerythritol) (XXX). Their concentrations were higher in summer than in winter (Table 1). The levels of 2-methyltetrols in the Chennai aerosols $\left(2.75 \pm 2.78 \mathrm{ng} \mathrm{m}^{-3}\right.$ in winter versus $13.8 \pm 9.10 \mathrm{ng} \mathrm{m}^{-3}$ in summer) are lower than those reported in aerosols from mountain sites in China (Wang et al., 2008; Fu et al., 2009c), and forested sites in Amazon (Claeys et al., 2004a), Hungary (Ion et al., 2005), Finland (Kourtchev et al., 2008a), and Germany (Kourtchev et al., 2008b). 2-Methylerythritol was about 2.5-fold more abundant than 2-methylthreitol (Table 1). This ratio is similar to those observed in other studies (Claeys et al., 2004a; Ion et al., 2005; Cahill et al., 2006). A strong correlation was found between 2-methylthreitol and 2methylerythritol $\left(R^{2}=0.99\right.$, Fig. $\left.6 \mathrm{~d}\right)$, suggesting a very similar formation pathway and/or atmospheric fate.

$\mathrm{C}_{5}$-Alkene triols, which are also photooxidation products of isoprene (Wang et al., 2005; Surratt et al., 2006), were detected in all the samples with average concentrations similar to those of 2-methyltetrols. However, they are lower than those $\left(\sim 50 \mathrm{ng} \mathrm{m}^{-3}\right)$ reported in subtropical Hong Kong (Hu et al., 2008), but are comparable to those reported in other studies from midlatitudes, e.g., a Californian pine forest, USA (3.47 $\mathrm{ng} \mathrm{m}^{-3}$ ) (Cahill et al., 2006), and Jülich, Germany (1.6-4.9 $\mathrm{ng} \mathrm{m}^{-3}$ ) (Kourtchev et al., 2008b), and about 2 orders of magnitude higher than those reported in the Arctic region (Fu et al., 2009b). A good correlation was found between 2-methyltetrols and $\mathrm{C}_{5}$-alkene triols in the Chennai samples (Fig. 6e). It is reasonable because they are both derived from the oxidation of isoprene. However, their formation processes are different. Wang et al. (2005) reported that 2-methyltetrols are formed through diepoxy derivatives of isoprene, which can be converted into 2-methyltetrols through acid-catalyzed hydrolysis. Alternatively, the formation of $\mathrm{C}_{5}$-alkene triols can be explained through rearrangement reactions of hydroxyperoxy radicals that are formed in the initial photooxidation of isoprene (Surratt et al., 2006). 2Methylglyceric acid is possibly formed by further oxidation of methacrolein and methacrylic acid from isoprene (Claeys et al., 2004b; Surratt et al., 2006). Its concentrations were lower than those of 2-methyltetrols and $\mathrm{C}_{5}$-alkene triols (Table 1).

Five organic acids were detected as $\alpha / \beta$-pinene oxidation products, including 3-hydroxyglutaric acid (3-HGA, XXXI), pinonic acid (XXXII), norpinic acid (XXXIII), pinic acid (XXXIV), and 3-methyl-1,2,3-butanetricarboxylic acid (MBTCA, XXXV). These acids are produced by the oxidation of $\alpha / \beta$-pinene via reactions with $\mathrm{O}_{3}$ and $\mathrm{OH}$ radicals (Hallquist et al., 2009). Concentrations of pinic and pinonic acids were similar during winter whereas in summer the concentrations of pinonic acid were higher than those of pinic acid (Table 1), although the vapor pressure of pinonic acid is about 2 orders of magnitude higher than pinic acid. This pattern is consistent with those found in tropospheric aerosols over Mt. Tai, in which pinonic acid was twice more abundant than pinic acid. Similar patterns have been reported in other studies (Kavouras et al., 1999; Cahill et al., 2006; Bhat and Fraser, 2007; Yan et al., 2008). However, higher concentrations of pinic acid than pinonic acid have been reported in the aerosols from a coniferous forest in Germany (Plewka et al., 2006) and Research Triangle Park (RTP), USA (Kleindienst et al., 2007). Both 3-HGA and MBTCA are generated in smog chamber experiments of $\alpha$-pinene with an irradiation of $\mathrm{UV}$ in the presence of $\mathrm{NO}_{\mathrm{x}}$ (Claeys et al., 2007; Szmigielski et al., 2007). 3-HGA was the most abundant species among the $\alpha / \beta$-pinene oxidation products.

$\beta$-Caryophyllinic acid (XXXVI), an ozonolysis or photooxidation product of $\beta$-caryophyllene (Jaoui et al., 2007), was identified at both winter and summer samples without any significant differences (Table 1). However, relatively higher levels of $\beta$-caryophyllinic acid were observed during late winter (Fig. 15q) when the air masses were originated from Southeast Asia and transported over the Bay of Bengal (Pavuluri et al., 2010), indicating that Southeast Asia is a strong "emitter" of $\beta$-caryophyllene.

Most of the biogenic SOA tracers gave diurnal trends with higher concentrations at daytime (Fig. 15), suggesting a photochemical production of these compounds. This pattern is different from those of biomass burning tracers (e.g., levoglucosan) and other anthropogenic organics such as hopanes and PAHs. The nighttime maxima of these organic compounds are mainly associated with the land/sea breeze circulation in Chennai. The temporal pattern of 2-methylglyceric acid (Fig. 15h) was similar to those of 2-methyltetrols (Fig. 15i) and $\mathrm{C}_{5}$-alkene triols (Fig. 15j). In addition, a clear diurnal variation can be found for 2methylglyceric acid during summer with higher concentrations at daytime. In winter, one concentration peak was observed during 30 January-1 February (Fig. $15 \mathrm{~h}-\mathrm{j}$ ). Such a peak can also be found for other SOA tracers and hydroxy/polyacids (Fig. 15). The temporal variations of $\alpha / \beta$-pinene oxidation tracers (Fig. 15k-p) were different from those of isoprene oxidation tracers (Fig. $15 \mathrm{~h}-\mathrm{j}$ ). In addition, the temporal patterns of pinonic acid and norpinic acid were different from those of pinic acid, 3-HGA and MBTCA. 3-HGA and MBTCA are later-generation photooxidation products of $\alpha / \beta$-pinene (Hallquist et al., 2009). These two species were well correlated each other $\left(R^{2}=0.67, n=49\right)$. In addition, both 3-HGA and MBTCA were well correlated with malic acid (Fig. 6f), indicating a similar formation pathway or similar source regions. The temporal variations of $\beta$-caryophyllinic acid (Fig. 15q) showed a clear diurnal pattern with daytime maxima. One concentration peak was observed during 30 January-1 February. This pattern was different from those of isoprene or $\alpha / \beta$-pinene SOA tracers.

Figure 16a shows the temporal trends of the total biogenic SOA tracers detected in the tropical Indian aerosols. In winter, $\alpha / \beta$-pinene oxidation products were the most abundant species, followed by isoprene and $\beta$-caryophyllene oxidation products. However in summer, isoprene oxidation products were found to be the most abundant, followed by $\alpha / \beta$-pinene 
Table 3. Contribution of individual organic compound classes to OC and WSOC in the aerosols from Chennai, tropical India (\%)

\begin{tabular}{|c|c|c|c|c|c|c|c|c|}
\hline \multirow{2}{*}{ Compound class } & \multicolumn{4}{|c|}{ Winter } & \multicolumn{4}{|c|}{ Summer } \\
\hline & $\min$ & $\max$ & average & $\mathrm{SD}^{\mathrm{b}}$ & $\min$ & $\max$ & average & $\mathrm{SD}$ \\
\hline \multicolumn{9}{|c|}{ To OC } \\
\hline$n$-alkanes & 0.25 & 2.39 & 1.18 & 0.55 & 0.50 & 3.72 & 1.37 & 0.91 \\
\hline UCM hydrocarbons & 1.05 & 10.6 & 5.00 & 2.61 & 1.89 & 16.8 & 5.98 & 4.17 \\
\hline fatty acids & 0.58 & 3.51 & 2.37 & 0.72 & 1.65 & 6.56 & 3.54 & 1.44 \\
\hline fatty alcohols & 0.17 & 0.90 & 0.62 & 0.14 & 0.36 & 0.85 & 0.57 & 0.14 \\
\hline anhydrosugars & 0.04 & 1.50 & 0.59 & 0.40 & 0.36 & 0.88 & 0.59 & 0.16 \\
\hline sugars/sugar alcohols & 0.02 & 0.34 & 0.10 & 0.07 & 0.05 & 0.28 & 0.14 & 0.05 \\
\hline lignin products & 0.01 & 0.23 & 0.12 & 0.05 & 0.05 & 0.11 & 0.08 & 0.02 \\
\hline terpenoid biomarkers & 0.02 & 0.13 & 0.06 & 0.03 & 0.02 & 0.05 & 0.03 & 0.01 \\
\hline sterols & 0.02 & 1.15 & 0.43 & 0.39 & 0.03 & 0.60 & 0.18 & 0.14 \\
\hline aromatic acids & 0.05 & 0.55 & 0.26 & 0.13 & 0.20 & 0.50 & 0.32 & 0.10 \\
\hline hydroxy-/polyacids & 0.02 & 0.72 & 0.16 & 0.15 & 0.08 & 0.36 & 0.20 & 0.09 \\
\hline biogenic SOA tracers & 0.01 & 0.43 & 0.11 & 0.10 & 0.05 & 0.54 & 0.24 & 0.12 \\
\hline plasticizers/antioxidants & 0.99 & 7.95 & 2.97 & 1.73 & 2.20 & 8.39 & 4.50 & 1.53 \\
\hline hopanes & 0.01 & 0.27 & 0.14 & 0.06 & 0.03 & 0.06 & 0.05 & 0.01 \\
\hline PAHs & 0.04 & 0.68 & 0.37 & 0.16 & 0.07 & 0.27 & 0.15 & 0.06 \\
\hline diacids and related compounds ${ }^{\mathrm{c}}$ & 0.77 & 7.26 & 2.98 & 1.74 & 0.85 & 2.87 & 1.80 & 0.58 \\
\hline Total Quantified & 11.0 & 24.2 & 17.5 & 3.37 & 13.6 & 32.8 & 19.7 & 5.21 \\
\hline \multicolumn{9}{|c|}{ To WSOC } \\
\hline sugars/sugar alcohols & 0.08 & 0.65 & 0.21 & 0.12 & 0.21 & 0.58 & 0.30 & 0.09 \\
\hline levoglucosan & 0.07 & 3.49 & 1.17 & 0.84 & 0.70 & 1.97 & 1.20 & 0.39 \\
\hline galactosan + mannosan & 0.01 & 0.64 & 0.23 & 0.16 & 0.09 & 0.29 & 0.17 & 0.06 \\
\hline hydroxy-/polyacids & 0.09 & 1.03 & 0.32 & 0.20 & 0.28 & 0.60 & 0.44 & 0.11 \\
\hline biogenic SOA tracers & 0.05 & 0.62 & 0.21 & 0.13 & 0.24 & 0.96 & 0.51 & 0.16 \\
\hline benzoic acid & 0.01 & 0.05 & 0.02 & 0.01 & 0.01 & 0.04 & 0.02 & 0.01 \\
\hline phthalic acid & 0.01 & 0.59 & 0.10 & 0.12 & 0.04 & 0.22 & 0.09 & 0.04 \\
\hline vanillic acid ${ }^{\mathrm{d}}$ & 0.001 & 0.031 & 0.015 & 0.009 & 0.007 & 0.017 & 0.011 & 0.003 \\
\hline 4-hydroxybenzoic acid ${ }^{\mathrm{d}}$ & 0.01 & 0.35 & 0.16 & 0.09 & 0.04 & 0.12 & 0.08 & 0.03 \\
\hline diacids and related compounds ${ }^{\mathrm{c}}$ & 3.63 & 11.5 & 6.37 & 2.64 & 2.58 & 5.91 & 3.92 & 0.72 \\
\hline Total Soluble & 5.10 & 13.0 & 8.80 & 2.07 & 5.82 & 8.44 & 6.75 & 0.61 \\
\hline
\end{tabular}

a OC, organic carbon; WSOC, water-soluble organic carbon. All the quantified organic compounds were converted to carbon contents to calculate the OC and WSOC ratios.

b Standard deviation.

c Including dicarboxylic acids $\left(\mathrm{C}_{2}-\mathrm{C}_{11}\right)$, ketoacids and $\alpha$-dicarbonyls that were reported by Pavuluri et al. (2010). Malic acid and phthalic acids were excluded because they have been reported in the present study.

d Slightly soluble in water.

and $\beta$-caryophyllene oxidation products. The total concentrations of biogenic SOA tracers were $18.3-107 \mathrm{ng} \mathrm{m}^{-3}$ (average $43.9 \mathrm{ng} \mathrm{m}^{-3}$ ) in summer versus $2.69-66.9 \mathrm{ng} \mathrm{m}^{-3}$ $\left(16.8 \mathrm{ng} \mathrm{m}^{-3}\right)$ in winter (Table 1). These values are lower than those from forested sites (Kourtchev et al., 2008a, b; Wang et al., 2008; Fu et al., 2009c), and urban sites in Hong Kong (Hu et al., 2008). This suggests that in tropical India, biogenic SOA tracers (e.g., 2-methyltetrols, 3-HGA and MBTCA) may be further oxidized to lower molecular weight compounds such as oxalic acid under strong sunlight radiation, a point warrants future study.
Contributions of isoprene, $\alpha / \beta$-pinene and $\beta$ caryophyllene oxidation products to OC were examined to better understand the chemical composition of SOA in tropical India. Mean contributions of total SOA tracers to OC in summer $(0.24 \pm 0.12 \%)$ were about twice higher than those in winter $(0.11 \pm 0.10 \%)$ (Table 3). Fig. $16 \mathrm{~b}$ shows the temporal contributions of isoprene, $\alpha / \beta$-pinene and $\beta$-caryophyllene oxidation products to $\mathrm{OC}$ with higher total percentages in summer when photochemical activity is high. Isoprene and $\alpha / \beta$-pinene SOA tracers contributed almost equally to $\mathrm{OC}$ in early winter. In late winter, the 

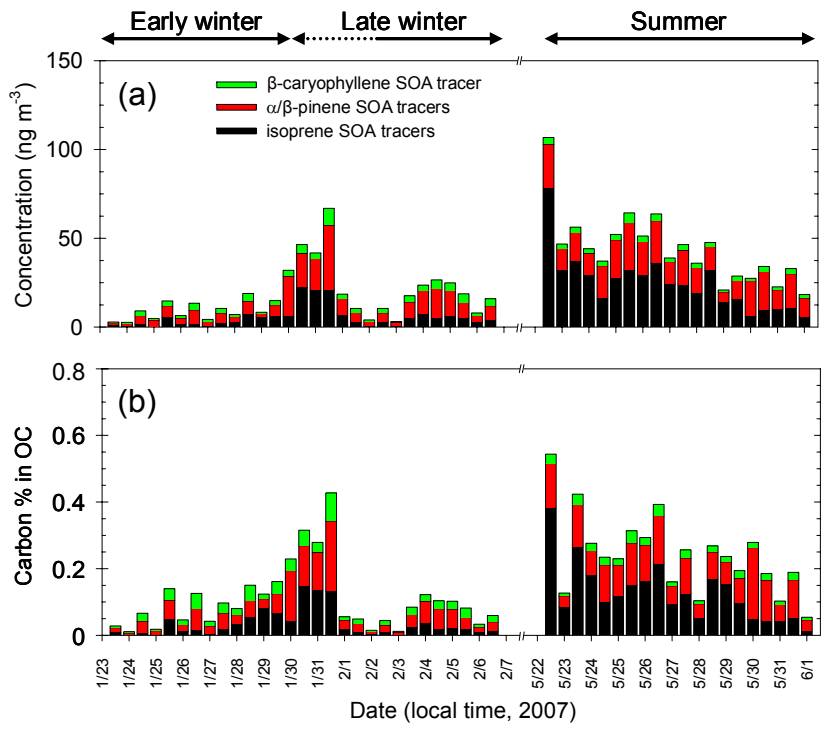

Fig. 16. Temporal variations of (a) the total concentrations of isoprene, $\alpha / \beta$-pinene and $\beta$-caryophyllene SOA tracers, and (b) the carbon percentage of biogenic SOA tracers in Organic Carbon (OC) in the tropical Indian aerosols.

contribution of $\alpha / \beta$-pinene SOA tracers to OC was found to be higher than those of isoprene SOA tracers. However in summer, the largest contributor is isoprene SOA tracers. The relative abundances of $\beta$-caryophyllene SOA tracer (i.e., $\beta$-caryophyllinic acid) in OC are similar in winter and summer (Fig. 16b), which suggests that there is no significant seasonal difference for the emissions of sesquiterpenes in tropical India. However in late winter when the air masses mainly originated from South Asia over the Bay of Bengal to the city of Chennai, the mean carbon $\%$ of $\beta$-caryophyllinic acid is comparable to those of isoprene SOA tracers.

\subsection{Source strengths of organic matter in the urban aerosols}

Based on the above discussion and previously studied emission profiles of organic compounds (Schauer et al., 1996; Simoneit et al., 2004), organic compound classes quantified in the Chennai aerosols, including Unresolved Complex Mixture (UCM) of branched and cyclic hydrocarbons, can be roughly apportioned to six emission sources and a group of secondary oxidation products as follows: (a) plant emission characterized by higher plant wax $n$-alkanes, HMW fatty acids and fatty alcohols; (b) microbial lipids or marine input mainly reflected from LMW fatty acids and fatty alcohols $\left(<\mathrm{C}_{20}\right)$; (c) biomass burning characterized by levoglucosan and its isomers, $\beta$-sitosterol, lignin products and terpenoid biomarkers; (d) fossil fuel combustion characterized by petroleum-derived $n$-alkanes, UCM, hopanes and PAHs; (e) soil resuspension characterized by primary saccharides and reduced sugars; (f) plastic emission including phthalates,

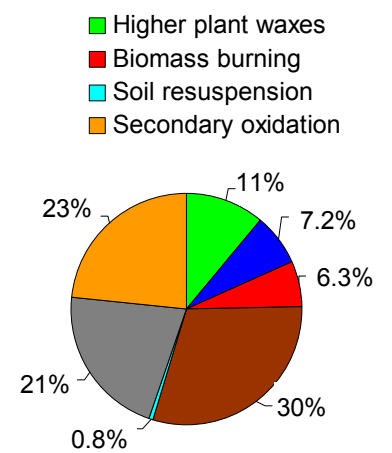

(a) Daytime (Winter)
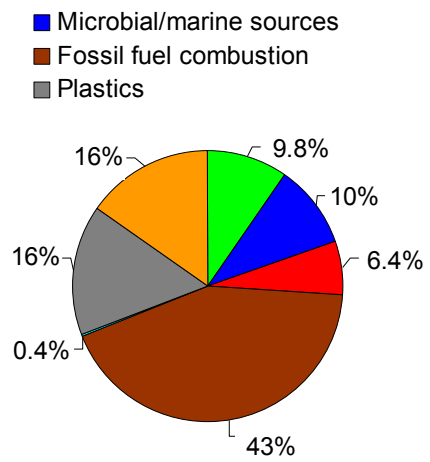

(b) Nighttime (Winter)

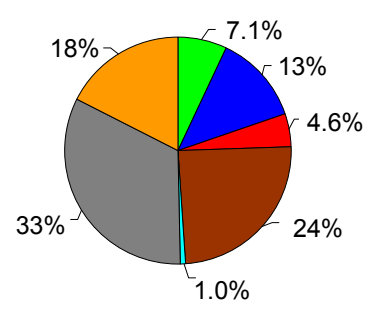

(c) Daytime (Summer)

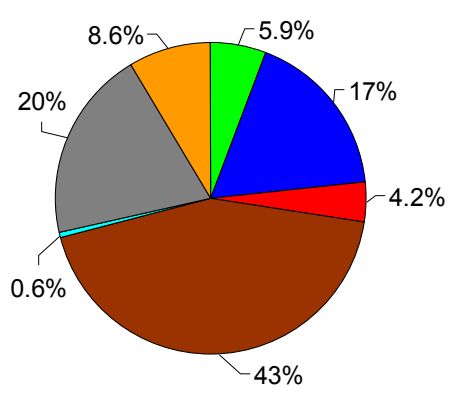

(d) Nighttime (Summer)
Fig. 17. Source strengths of organic matter in the urban aerosols from Chennai, tropical India. The sizes of the pie diagrams are proportional to the average ambient $\mathrm{OC}$ concentrations during each period.

TBPP, terephthalic acid, 1,3,5-triphenylbenzene and 1,2,4triphenylbenzene; and $(\mathrm{g})$ secondary oxidation products characterized by aromatic acids, hydroxy-/polyacids, biogenic SOA tracers, and dicarboxylic acids and related compounds.

The source strengths are apportioned based on organic tracers for selected samples, as shown in Fig. 17. In winter, the most abundant group was fossil fuel combustion products $(30-43 \%)$, followed by secondary oxidation products, plastics and higher plant waxes. During the summer daytime, plastic emission was the most significant source to the organic aerosols, followed by fossil fuel combustion and secondary oxidation. During the summer nighttime, fossil fuel combustion was the most important source, followed by plastic emission and microbial/marine sources. Secondary oxidation products are more important at daytime than nighttime for both winter and summer seasons. However, their contributions to OC were higher in winter $(23 \%$ at daytime versus $16 \%$ at nighttime) than summer (18\% versus $8.6 \%$ ). The abundant secondary oxidation products in urban aerosols is equivalent to the biomass burning tracers, suggesting that biomass burning may enhance the formation of SOA in winter. Grieshop et al. (2009) reported that SOA can be efficiently formed from gas-phase precursors in biomass burning emissions. It should be noted that this 
source apportionment approach is useful to roughly estimate the relative contribution from different emission sources to the organic aerosols, because some organic species may come from multiple sources, and thus a potential overlapping problem cannot be excluded (Simoneit et al., 2004).

\subsection{Contributions to $\mathrm{OC}$ and WSOC}

Contributions of each compound class to OC and WSOC in the samples were examined (Table 3). Sugar compounds (including anhydrosugars and sugar alcohols) accounted for $0.09-1.67 \%(0.69 \%)$ of OC in winter and 0.40 $1.06 \%(0.73 \%)$ of OC in summer. They are lower than those reported in urban aerosols from Hong Kong (0.3-3.6\%, average 1.3\%) (Wan and Yu, 2007). Although anhydrosugars comprise a similar fraction in OC (average $0.59 \%$ in winter and summer), contributions of other biomass burning tracers such as lignin products, terpenoid biomarkers, and sterols to OC are 2-3 times higher in winter than in summer. This indicates that intensive biomass/biofuel burning can more significantly affect the chemical composition of organic aerosols in tropical India during winter. In contrast, summertime samples showed more contributions of fatty acids and aromatic acids to OC. This suggests that both biological emission and photochemical production are more important in summer than winter. Similarly, plastic-derived products showed higher contribution to OC in summer due to a fugitive evaporation of plasticizers. UCM hydrocarbons of fossil fuel combustion origin are found to compose 1.05$10.6 \%$ (average $5.00 \%$ ) of aerosol OC in winter and 1.89 $16.8 \%(5.98 \%)$ of OC in summer. These values are higher than those (1.0-6.6\%, average 3.4\%) reported in Sapporo, Japan, and are much higher than those $(0.37-1.5 \%$, average $0.86 \%$ ) in Chichi-Jima, a remote island in the western North Pacific (Simoneit et al., 2004). With the consideration of dicarboxylic acids and related compounds (ketoacids and $\alpha$-dicarbonyls) that were detected in the same sample sets as reported in Pavuluri et al. (2010), the total organics identified in the tropical Indian aerosols accounted for $17.5 \pm 3.37 \%$ of OC in winter versus $19.7 \pm 5.21 \%$ in summer with a maximum of $32.8 \%$ (Table 3 ). This indicates that approximately one third of organic aerosols studied in the tropical Indian region can be identified at a molecular level.

The percentage of levoglucosan in WSOC ranged from $0.07-3.49 \%$ (average $1.17 \%$ ) in winter versus $0.70-1.97 \%$ $(1.20 \%)$ in summer. These values are comparative to those reported in Mt. Tai aerosols in Central East China (average $1.50 \%$ at daytime versus $1.13 \%$ at nighttime) (Fu et al., 2008). The contributions of sugars/sugar alcohols to WSOC were $0.21 \pm 0.12 \%$ in winter and $0.30 \pm 0.09 \%$ in summer. The higher contribution of primary saccharides to WSOC in summer than in winter indicates that atmospheric sugar compounds may be associated with an enhanced emission of primary bioaerosols or soil resuspension in hot season. Biogenic SOA tracers accounted for $0.51 \pm 0.16 \%$ of WSOC in summer, which double those in winter $(0.21 \pm 0.13 \%)$. This again suggests that the photochemical production of SOA is more important in summer than in winter. Total watersoluble organics detected in the Chennai aerosols accounted for $8.80 \pm 2.07 \%$ and $6.75 \pm 0.61 \%$ of WSOC in winter and summer, respectively. It is of interest to note that the major species contributed to WSOC are small dicarboxylic acids (Table 3), which have been reported as dominant organic compounds in atmospheric aerosols from different locations in the world (Kawamura and Ikushima, 1993; Kawamura et al., 1996; Kawamura and Sakaguchi, 1999).

\section{Conclusions}

Concentrations of total quantified organic compounds, together with UCM hydrocarbons in the tropical Indian aerosols were higher in summer $\left(780-6607 \mathrm{ng} \mathrm{m}^{-3}\right.$, average $2506 \mathrm{ng} \mathrm{m}^{-3}$ ) than in winter (436-4399 $\mathrm{ng} \mathrm{m}^{-3}$, $1800 \mathrm{ng} \mathrm{m}^{-3}$ ). With the consideration of dicarboxylic acids and related compounds (ketoacids and $\alpha$-dicarbonyls), the total identified organics accounted for 11.0-24.2\% (average $17.5 \%)$ and $13.6-32.8 \%$ (19.7\%) of OC in winter and summer, respectively. Although these values may be underestimated due to a potential evaporative loss of semivolatile compounds, this study suggests that the major portion $(70-80 \%)$ of organic aerosols is still not specified in terms of molecular levels, which may include humic-like substances, oligmers, organosulfates, organic nitrates, and others (Kanakidou et al., 2005; Graber and Rudich, 2006; Goldstein and Galbally, 2007; Hallquist et al., 2009).

The abundances of anhydrosugars, lignin products, terpenoid biomarkers, sterols, hopanes, and PAHs suggest that both biomass/biofuel burning and fossil fuel combustion are important primary sources of organic aerosols in tropical India. Microbial/marine sources also contribute to primary organic aerosols in tropical India, especially in summer. Meanwhile, high abundances of 1,3,5-triphenylbenzene and tris(2,4-di-tert-butyl-phenyl)phosphate, together with prevailing concentrations of terephthalic acid at nighttime than daytime, suggest that the open burning of municipal solid wastes including plastics is also a significant source for primary atmospheric aerosols in this region. These anthropogenic particles in South Asia should cause further environmental concerns. Secondary products derived from the oxidation of biogenic VOCs and other organic precursors, for example, from biomass burning also contribute significantly to organic aerosols. They consist of isoprene, $\alpha / \beta$ pinene and $\beta$-caryophyllene SOA tracers, dicarboxylic acids and polyacids, aromatic acids, hydroxyacids etc. Such information may be helpful to better understand the sources and formation processes of atmospheric brown clouds in South Asia. 


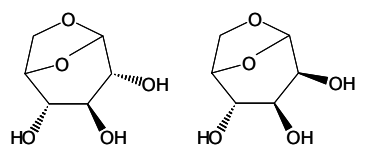

I. Levoglucosan II. Mannosan<smiles>COc1cc(CC2COCC2Cc2ccc(O)c(OC)c2)ccc1O</smiles>
VIII. Shonanin<smiles>C=C[C@]1(C)CCC2C(=CCC3[C@@](C)(C(=O)O)CCC[C@]23C)C1</smiles>

XIII.Iso-pimaric acid<smiles>C[AsH3]OC([GeH3])[GeH3]</smiles>

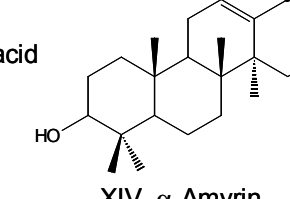

IX. Dehydroabietic acid
$\sum_{\mathrm{OH}}^{\mathrm{O}}$

III. Galactosan<smiles>O=C(O)c1ccc(O)cc1</smiles>

IV. 4-Hydroxybenzoic acid<smiles>O=C(O)c1ccc(O)c(O)c1</smiles>

V. Vanillic acid<smiles>O=Cc1ccc(O)c(O)c1</smiles>

VI. Vanillin<smiles>O=C(O)c1cc(O)c(O)c(O)c1</smiles>

VII. Syringic acid<smiles>CC(C)c1ccc2c(c1)CC[C@]1(C)[C@@H](C(=O)O)CCC[C@@]21C</smiles>

X. 7-Oxodehydroabietic acid<smiles>CC(C)C1=CC2=CC[C@H]3C(C)(C)CCCC3(C)C2CC1</smiles>

XI. Abietic acid

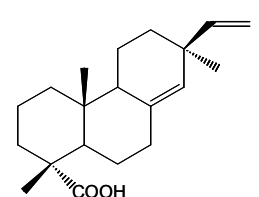

XII. Pimaric acid

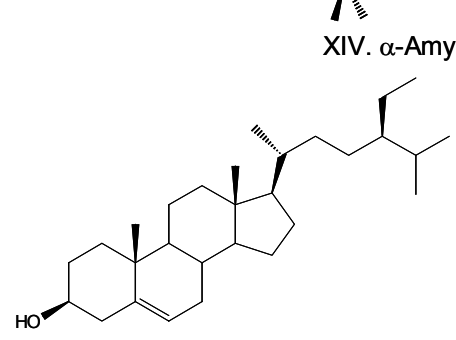
XVIII. $\beta$-Sitosterol XIX. Di-(2-ethylhexyl) phthalate
(DEHP) XIX. Di-(2-ethylhexy)
(DEHP)<smiles>CCCCC(CC)COC(=O)c1ccccc1C(=O)OCC(CC)CCCC</smiles><smiles>c1ccc(-c2ccc(-c3ccccc3)c(-c3ccccc3)c2)cc1</smiles>

XXII. 1,2,4-Triphenylbenzene<smiles>[R]C(C)C1CCC2C1CCC1C2CCC2C(C)C3(C)CCCC(C)(C)C3CCC12CCCCCC</smiles>

XXIII. $17 \alpha(H)$-Hopanes<smiles>C/C(=C/O)C(O)CO</smiles>

XXVII. Cis-2-methyl-1,3,4trihydroxy-1-butene<smiles>CC(=O)C1CC(CC(=O)O)C1(C)C</smiles>

XXXII. Pinonic acid<smiles>C=C(O)C(C)(O)CO</smiles>

XXVIII. 3-Methyl-2,3,4trihydroxy-1-butene

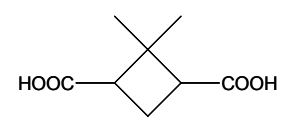

XXXIII. Norpinic acid<smiles>CC(=CO)C(O)CO</smiles>

XXIX. Trans-2-methyl-1,3,4trihydroxy-1-butene

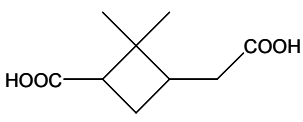

XXXIV. Pinic acid
XVII. Stigmasterol

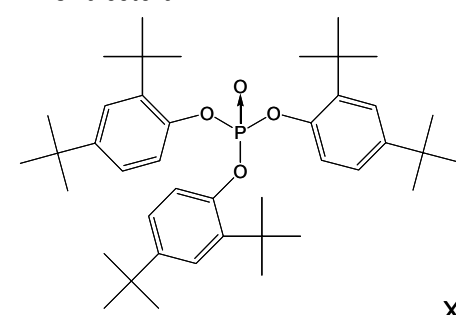

XX. Tris(2,4-di-tert-butyl-phenyl)phosphate

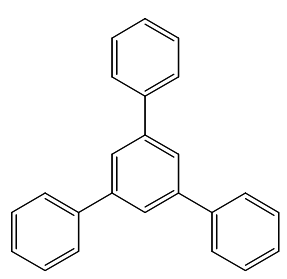

XXI. 1,3,5-Triphenylbenzene<smiles>OCC(O)C(O)CO</smiles>

XXIV. Erythritol

HOH

$X X V$. Sucrose
$\overbrace{\mathrm{OH}}^{\mathrm{HOOOH}}$

XXVI. 2-Methylglyceric acid<smiles>CC(O)(CO)C(O)CO</smiles><smiles>O=C(O)CC(O)CC(=O)O</smiles>

XXXI. 3-Hydroxyglutaric acid

XXX. 2-Methyltetrols<smiles>CC(C)(C(=O)O)C(CC(=O)O)C(=O)O</smiles>

XXXV. MBTCA

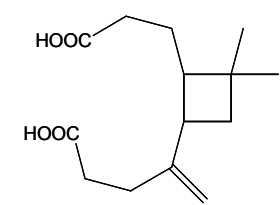

XXXVI. $\beta$-Caryophyllinic acid

Fig. A1. Chemical structures cited in the text. 
Acknowledgements. This study is partly supported by the Japanese Ministry of Education, Culture, Sports, Science and Technology (MEXT) through grant-in-aid nos. 17340166 and 19204055. P. F. appreciates the Japan Society for the Promotion of Science (JSPS) for a research fellowship. We also thank R. A. Damodar and K. Jagannathan for their help during the sample collection.

Edited by: R. Holzinger

\section{References}

Alves, C., Pio, C., and Duarte, A.: Composition of extractable organic matter of air particles from rural and urban Portuguese areas, Atmos. Environ., 35, 5485-5496, 2001.

Andreae, M. O. and Crutzen, P. J.: Atmospheric aerosols: Biogeochemical sources and role in atmospheric chemistry, Science, 276, 1052-1058, 1997.

Andreae, M. O. and Rosenfeld, D.: Aerosol-cloud-precipitation interactions. Part 1, The nature and sources of cloud-active aerosols, Earth-Sci. Rev., 89, 13-41, 2008.

Bauer, H., Claeys, M., Vermeylen, R., Schueller, E., Weinke, G., Berger, A., and Puxbaum, H.: Arabitol and mannitol as tracers for the quantification of airborne fungal spores, Atmos. Environ., 42, 588-593, 2008.

Bhat, S. and Fraser, M. P.: Primary source attribution and analysis of $\alpha$-pinene photooxidation products in Duke Forest, North Carolina, Atmos. Environ., 41, 2958-2966, 2007.

Bieleski, R. L.: Onset of phloem export from senescent petals of daylily, Plant Physiol., 109, 557-565, 1995.

Bin Abas, M. R., Simoneit, B. R. T., Elias, V., Cabral, J. A., and Cardoso, J. N.: Composition of higher molecular weight organic matter in smoke aerosol from biomass combustion in Amazonia, Chemosphere, 30, 995-1015, 1995.

Cahill, T. M., Seaman, V. Y., Charles, M. J., Holzinger, R., and Goldstein, A. H.: Secondary organic aerosols formed from oxidation of biogenic volatile organic compounds in the Sierra Nevada Mountains of California, J. Geophys. Res.-Atmos., 111, D16312, doi:10.1029/2006JD007178, 2006.

Chowdhury, Z., Zheng, M., Schauer, J. J., Sheesley, R. J., Salmon, L., Cass, G. R., and Russell, A.: Speciation of ambient fine organic carbon particles and source apportionment of $\mathrm{PM}_{2.5}$ in Indian cities, J. Geophys. Res.-Atmos., 112, D15303, doi:10.1029/2007JD008386, 2007.

Claeys, M., Graham, B., Vas, G., Wang, W., Vermeylen, R., Pashynska, V., Cafmeyer, J., Guyon, P., Andreae, M. O., Artaxo, P., and Maenhaut, W.: Formation of secondary organic aerosols through photooxidation of isoprene, Science, 303, 1173-1176, 2004a.

Claeys, M., Wang, W., Ion, A. C., Kourtchev, I., Gelencsér, A., and Maenhaut, W.: Formation of secondary organic aerosols from isoprene and its gas-phase oxidation products through reaction with hydrogen peroxide, Atmos. Environ., 38, 4093-4098, 2004b.

Claeys, M., Szmigielski, R., Kourtchev, I., van der Veken, P., Vermeylen, R., Maenhaut, W., Jaoui, M., Kleindienst, T. E., Lewandowski, M., Offenberg, J., and Edney, E. O.: Hydroxydicarboxylic acids: Markers for secondary organic aerosol from the photooxidation of $\alpha$-pinene, Environ. Sci. Technol., 41, 16281634, 2007.
Crutzen, P. J. and Andreae, M. O.: Biomass burning in the tropics: Impact on atmospheric chemistry and biogeochemical cycles, Science, 250, 1669-1678, 1990.

de Gouw, J. and Jimenez, J. L.: Organic aerosols in the Earth's atmosphere, Environ. Sci. Technol., 43, 7614-7618, 2009.

Duhl, T. R., Helmig, D., and Guenther, A.: Sesquiterpene emissions from vegetation: a review, Biogeosciences, 5, 761-777, 2008, http://www.biogeosciences.net/5/761/2008/.

Forstner, H. J. L., Flagan, R. C., and Seinfeld, J. H.: Secondary organic aerosol from the photooxidation of aromatic hydrocarbons: Molecular composition, Environ. Sci. Technol., 31, 1345-1358, 1997.

Fraser, M. P., Cass, G. R., and Simoneit, B. R. T.: Gas-phase and particle-phase organic compounds emitted from motor vehicle traffic in a Los Angeles roadway tunnel, Environ. Sci. Technol., 32, 2051-2060, 1998.

Fraser, M. P., Cass, G. R., and Simoneit, B. R. T.: Air quality model evaluation data for organics, $6 . \mathrm{C}_{3}-\mathrm{C}_{24}$ organic acids, Environ. Sci. Technol., 37, 446-453, 2003.

Fu, P. Q., Kawamura, K., Okuzawa, K., Aggarwal, S. G., Wang, G., Kanaya, Y., and Wang, Z.: Organic molecular compositions and temporal variations of summertime mountain aerosols over Mt. Tai, North China Plain, J. Geophys. Res.-Atmos., 113, D19107, doi:10.1029/2008JD009900, 2008.

Fu, P. Q., Kawamura, K., and Barrie, L. A.: Photochemical and other sources of organic compounds in the Canadian high Arctic aerosol pollution during winter-spring, Environ. Sci. Technol., 43, 286-292, 2009a.

Fu, P. Q., Kawamura, K., Chen, J., and Barrie, L. A.: Isoprene, monoterpene, and sesquiterpene oxidation products in the high Arctic aerosols during late winter to early summer, Environ. Sci. Technol., 43, 4022-4028, 2009b.

Fu, P. Q., Kawamura, K., Pochanart, P., Tanimoto, H., Kanaya, Y., and Wang, Z. F.: Summertime contributions of isoprene, monoterpenes, and sesquiterpene oxidation to the formation of secondary organic aerosol in the troposphere over Mt. Tai, Central East China during MTX2006, Atmos. Chem. Phys. Discuss., 9, 16941-16972, 2009c, http://www.atmos-chem-phys-discuss.net/9/16941/2009/.

Gagosian, R. B., Zafiriou, O. C., Peltzer, E. T., and Alford, J. B.: Lipid in aerosols from the tropical North Pacific: Temporal variability, J. Geophys. Res., 87, 11133-11144, 1982.

Goldstein, A. H. and Galbally, I. E.: Known and unexplored organic constituents in the Earth's atmosphere, Environ. Sci. Technol., 41, 1514-1521, 2007.

Graber, E. R. and Rudich, Y.: Atmospheric HULIS: How humiclike are they? A comprehensive and critical review, Atmos. Chem. Phys., 6, 729-753, 2006, http://www.atmos-chem-phys.net/6/729/2006/.

Graham, B., Mayol-Bracero, O. L., Guyon, P., Robert, G. C., Decesari, S., Facchini, M. C., Artaxo, P., Maenhaut, W. P. K., and Andreae, M. O.: Water-soluble organic compounds in biomass burning aerosols over Amazonia: 1. Characterization by NMR and GC-MS, J. Geophys. Res., 107(D20), 8047, doi:8010.1029/2001JD000336, 2002.

Graham, B., Guyon, P., Taylor, P. E., Artaxo, P., Maenhaut, W., Glovsky, M. M., Flagan, R. C., and Andreae, M. O.: Organic compounds present in the natural Amazonian aerosol: Characterization by gas chromatography-mass spectrometry, J. Geophys. 
Res.-Atmos., 108(D24), 4766, doi:4710.1029/2003JD003990, 2003.

Grieshop, A. P., Logue, J. M., Donahue, N. M., and Robinson, A. L.: Laboratory investigation of photochemical oxidation of organic aerosol from wood fires 1: measurement and simulation of organic aerosol evolution, Atmos. Chem. Phys., 9, 1263-1277, 2009 ,

http://www.atmos-chem-phys.net/9/1263/2009/.

Guenther, A., Karl, T., Harley, P., Wiedinmyer, C., Palmer, P. I., and Geron, C.: Estimates of global terrestrial isoprene emissions using MEGAN (Model of Emissions of Gases and Aerosols from Nature), Atmos. Chem. Phys., 6, 3181-3210, 2006, http://www.atmos-chem-phys.net/6/3181/2006/.

Gustafsson, Ö., Kruså, M., Zencak, Z., Sheesley, R. J., Granat, L., Engström, E., Praveen, P. S., Rao, P. S. P., Leck, C., and Rodhe, H.: Brown clouds over South Asia: Biomass or fossil fuel combustion?, Science, 323, 495-498, 2009.

Hallquist, M., Wenger, J. C., Baltensperger, U., Rudich, Y., Simpson, D., Claeys, M., Dommen, J., Donahue, N. M., George, C., Goldstein, A. H., Hamilton, J. F., Herrmann, H., Hoffmann, T., Iinuma, Y., Jang, M., Jenkin, M. E., Jimenez, J. L., Kiendler-Scharr, A., Maenhaut, W., McFiggans, G., Mentel, Th. F., Monod, A., Prévôt, A. S. H., Seinfeld, J. H., Surratt, J. D., Szmigielski, R., and Wildt, J.: The formation, properties and impact of secondary organic aerosol: current and emerging issues, Atmos. Chem. Phys., 9, 5155-5236, 2009, http://www.atmos-chem-phys.net/9/5155/2009/.

Hartz, K. E. H., Tischuk, J. E., Chan, M. N., Chan, C. K., Donahue, N. M., and Pandis, S. N.: Cloud condensation nuclei activation of limited solubility organic aerosol, Atmos. Environ., 40, 605617,2006

Hoffmann, T., Odum, J. R., Bowman, F., Collins, D., Klockow, D., Flagan, R. C., and Seinfeld, J. H.: Formation of organic aerosols from the oxidation of biogenic hydrocarbons, J. Atmos. Chem., 26, 189-222, 1997.

Hopke, P. K.: Receptor Modeling in Environmental Chemistry, John Wiley \& Sons, New York, 1985.

Hu, D., Bian, Q., Li, T. W. Y., Lau, A. K. H., and Yu, J. Z.: Contributions of isoprene, monoterpenes, $\beta$-caryophyllene, and toluene to secondary organic aerosols in Hong Kong during the summer of 2006, J. Geophys. Res.-Atmos., 113, D22206, doi:10.1029/2008JD010437, 2008.

Ion, A. C., Vermeylen, R., Kourtchev, I., Cafmeyer, J., Chi, X., Gelencsër, A., Maenhaut, W., and Claeys, M.: Polar organic compounds in rural $\mathrm{PM}_{2.5}$ aerosols from K-puszta, Hungary, during a 2003 summer field campaign: Sources and diel variations, Atmos. Chem. Phys., 5, 1805-1814, 2005,

http://www.atmos-chem-phys.net/5/1805/2005/.

Jaoui, M., Lewandowski, M., Kleindienst, T. E., Offenberg, J. H., and Edney, E. O.: $\beta$-Caryophyllinic acid: An atmospheric tracer for $\beta$-caryophyllene secondary organic aerosol, Geophys. Res. Lett., 34, L05816, doi:10.1029/2006GL028827, 2007.

Kanakidou, M., Seinfeld, J. H., Pandis, S. N., Barnes, I., Dentener, F. J., Facchini, M. C., Van Dingenen, R., Ervens, B., Nenes, A., Nielsen, C. J., Swietlicki, E., Putaud, J. P., Balkanski, Y., Fuzzi, S., Horth, J., Moortgat, G. K., Winterhalter, R., Myhre, C. E. L., Tsigaridis, K., Vignati, E., Stephanou, E. G., and Wilson, J.: Organic aerosol and global climate modelling: a review, Atmos. Chem. Phys., 5, 1053-1123, 2005, http://www.atmos-chem-phys.net/5/1053/2005/.

Kavouras, I. G., Mihalopoulos, N., and Stephanou, E. G.: Formation and gas/particle partitioning of monoterpenes photooxidation products over forests, Geophys. Res. Lett., 26, 55-58, 1999.

Kawamura, K. and Gagosian, R. B.: Implications of $\omega$ oxocarboxylic acids in the remote marine atmosphere for photooxidation of unsaturated fatty acids, Nature, 325, 330-332, 1987.

Kawamura, K. and Kaplan, I. R.: Motor exhaust emissions as a primary source for dicarboxylic acids in Los Angels ambient air, Environ. Sci. Technol., 21, 105-110, 1987.

Kawamura, K. and Ikushima, K.: Seasonal changes in the distribution of dicarboxylic acids in the urban atmosphere, Environ. Sci Technol., 27, 2227-2235, 1993.

Kawamura, K., Kosaka, M., and Sempéré, R.: Distributions and seasonal changes of hydrocarbons in urban aerosols and rainwaters, Chikyukagaku, Geochemistry, 29, 1-15, 1995.

Kawamura, K., Kasukabe, H., and Barrie, L. A.: Source and reaction pathways of dicarboxylic acids, ketoacids and dicarbonyls in arctic aerosols: One year of observations, Atmos. Environ., 30, 1709-1722, 1996.

Kawamura, K. and Sakaguchi, F.: Molecular distributions of water soluble dicarboxylic acids in marine aerosols over the $\mathrm{Pa}$ cific Ocean including tropics, J. Geophys. Res.-Atmos., 104(D3), 3501-3509, 1999.

Kawamura, K., Steinberg, S., and Kaplan, I. R.: Homologous series of $\mathrm{C}_{1}-\mathrm{C}_{10}$ monocarboxylic acids and $\mathrm{C}_{1}-\mathrm{C}_{6}$ carbonyls in Los Angeles air and motor vehicle exhausts, Atmos. Environ., 34, 4175-4191, 2000.

Kawamura, K., Ishimura, Y., and Yamazaki, K.: Four years' observations of terrestrial lipid class compounds in marine aerosols from the western North Pacific, Global Biogeochem. Cy., 17, 1003, doi:1010.1029/2001GB001810, 2003.

Kleindienst, T. E., Jaoui, M., Lewandowski, M., Offenberg, J. H., Lewis, C. W., Bhave, P. V., and Edney, E. O.: Estimates of the contributions of biogenic and anthropogenic hydrocarbons to secondary organic aerosol at a southeastern US location, Atmos. Environ., 41, 8288-8300, 2007.

Kourtchev, I., Ruuskanen, T. M., Keronen, P., Sogacheva, L., Dal Maso, M., Reissell, A., Chi, X., Vermeylen, R., Kulmala, M., Maenhaut, W., and Claeys, M.: Determination of isoprene and $\alpha-/ \beta$-pinene oxidation products in boreal forest aerosols from Hyytiälä, Finland: diel variations and possible link with particle formation events, Plant Biol., 10, 138-149, 2008a.

Kourtchev, I., Warnke, J., Maenhaut, W., Hoffmann, T., and Claeys, M.: Polar organic marker compounds in $\mathrm{PM}_{2.5}$ aerosol from a mixed forest site in western Germany, Chemosphere, 73, 13081314, 2008b.

Lelieveld, J., Crutzen, P. J., Ramanathan, V., Andreae, M. O., Brenninkmeijer, C. A. M., Campos, T., Cass, G. R., Dickerson, R. R., Fischer, H., de Gouw, J. A., Hansel, A., Jefferson, A., Kley, D., de Laat, A. T. J., Lal, S., Lawrence, M. G., Lobert, J. M., MayolBracero, O. L., Mitra, A. P., Novakov, T., Oltmans, S. J., Prather, K. A., Reiner, T., Rodhe, H., Scheeren, H. A., Sikka, D., and Williams, J.: The Indian Ocean Experiment: Widespread air pollution from South and Southeast Asia, Science, 291, 1031-1036, 2001.

Matsunaga, S., Kawamura, K., Nakatsuka, T., and Ohkouchi, N.: Preliminary study on laboratory photochemical formation of low 
molecular weight dicarboxylic acids from unsaturated fatty acid (oleic acid), Res. Org. Geochem., 14, 19-25, 1999.

Medeiros, P. M., Conte, M. H., Weber, J. C., and Simoneit, B. R. T.: Sugars as source indicators of biogenic organic carbon in aerosols collected above the Howland Experimental Forest, Maine, Atmos. Environ., 40, 1694-1705, 2006.

Medeiros, P. M. and Simoneit, B. R. T.: Source profiles of organic compounds emitted upon combustion of green vegetation from temperate climate forests, Environ. Sci. Technol., 42, 83108316, 2008.

Mochida, M., Kitamori, Y., Kawamura, K., Nojiri, Y., and Suzuki, K.: Fatty acids in the marine atmosphere: Factors governing their concentrations and evaluation of organic films on sea-salt particles, J. Geophys. Res.-Atmos., 107(D17), 4325, doi:4310.1029/2001JD001278, 2002.

Mochida, M., Kawabata, A., Kawamura, K., Hatsushika, H., and Yamazaki, K.: Seasonal variation and origins of dicarboxylic acids in the marine atmosphere over the western North Pacific, J. Geophys. Res.-Atmos., 108(D6), 4193, doi:4110.1029/2002JD002355, 2003.

Nakajima, H., Okada, K., Kuroki, Y., Nakama, Y., Handa, D., Arakaki, T., and Tanahara, A.: Photochemical formation of peroxides and fluorescence characteristics of the water-soluble fraction of bulk aerosols collected in Okinawa, Japan, Atmos. Environ., 42, 3046-3058, 2008.

Nolte, C. G., Schauer, J. J., Cass, G. R., and Simoneit, B. R. T.: Highly polar organic compounds present in wood smoke and in the ambient atmosphere, Environ. Sci. Technol., 35, 1912-1919, 2001.

Oros, D. R. and Simoneit, B. R. T.: Identification and emission factors of molecular tracers in organic aerosols from biomass burning Part 2, Deciduous trees, Appl. Geochem., 16, 1545-1565, 2001.

Pöschl, U.: Atmospheric aerosols: Composition, transformation, climate and health effects, Angew. Chem. Int. Edit., 44, 75207540, 2005.

Pavuluri, C. M., Kawamura, K., and Swaminathan, T.: Watersoluble organic carbon, dicarboxylic acids, ketoacids and $\alpha$ dicarbonyls in the tropical Indian aerosols, J. Geophys. Res.Atmos., 115, doi:10.1029/2009JD012661, in press, 2010.

Plewka, A., Gnauk, T., Bruggemann, E., and Herrmann, H.: Biogenic contributions to the chemical composition of airborne particles in a coniferous forest in Germany, Atmos. Environ., 40, S103-S115, 2006.

Ramanathan, V., Chung, C., Kim, D., Bettge, T., Buja, L., Kiehl, J. T., Washington, W. M., Fu, Q., Sikka, D. R., and Wild, M.: Atmospheric brown clouds: Impacts on South Asia climate and hydrological cycle, P. Natl. Acad. Sci., 102, 5326-5333, 2005.

Robinson, A. L., Donahue, N. M., and Rogge, W. F.: Photochemical oxidation and changes in molecular composition of organic aerosol in the regional context, J. Geophys. Res.-Atmos., 111, D03302, doi:10.1029/2005JD006265, 2006.

Robinson, A. L., Donahue, N. M., Shrivastava, M. K., Weitkamp, E. A., Sage, A. M., Grieshop, A. P., Lane, T. E., Pierce, J. R., and Pandis, S. N.: Rethinking organic aerosols: Semivolatile emissions and photochemical aging, Science, 315, 1259-1262, 2007.

Rogge, W. F., Hildemann, L. M., Mazurek, M. A., Cass, G. R., and Simoneit, B. R. T.: Source of fine organic aerosols: 1. Charbroilers and meat cooking operations, Environ. Sci. Technol., 25,
1112-1125, 1991.

Rogge, W. F., Hildemann, L. M., Mazurek, M. A., Cass, G. R., and Simoneit, B. R. T.: Sources of fine organic aerosol, 2. Noncatalyst and catalyst-equipped automobiles and heavy-duty diesel trucks, Environ. Sci. Technol., 27, 636-651, 1993.

Rogge, W. F., Hildemann, L. M., Mazurek, M. A., Cass, G. R., and Simoneit, B. R. T.: Mathematical modeling of atmospheric fine particle-associated primary organic compound concentrations, J. Geophys. Res.-Atmos., 101(D14), 19379-19394, 1996.

Rudich, Y., Donahue, N. M., and Mentel, T. F.: Aging of organic aerosol: Bridging the gap between laboratory and field studies, Annu. Rev. Phys. Chem., 58, 321-352, 2007.

Schauer, J. J., Rogge, W. F., Hildemann, L. M., Mazurek, M. A., and Cass, G. R.: Source apportionment of airborne particulate matter using organic compounds as tracers, Atmos. Environ., 30, 3837-3855, 1996.

Schauer, J. J., Kleeman, M. J., Cass, G. R., and Simoneit, B. R. T.: Measurement of emissions from air pollution sources, 2. $\mathrm{C}_{1}$ through $\mathrm{C}_{30}$ organic compounds from medium duty diesel trucks, Environ. Sci. Technol., 33, 1578-1587, 1999.

Schauer, J. J., Kleeman, M. J., Cass, G. R., and Simoneit, B. R. T.: Measurement of emissions from air pollution sources, 3. $\mathrm{C}_{1}-\mathrm{C}_{29}$ organic compounds from fireplace combustion of wood, Environ. Sci. Technol., 35, 1716-1728, 2001.

Schauer, J. J., Kleeman, M. J., Cass, G. R., and Simoneit, B. R. T.: Measurement of emissions from air pollution sources, 5. $\mathrm{C}_{1^{-}}$ $\mathrm{C}_{32}$ organic compounds from gasoline-powered motor vehicles, Environ. Sci. Technol., 36, 1169-1180, 2002.

Seinfeld, J. H.: Black carbon and brown clouds, Nat. Geosci., 1, 15-16, 2008.

Sheesley, R. J., Schauer, J. J., Chowdhury, Z., Cass, G. R., and Simoneit, B. R. T.: Characterization of organic aerosols emitted from the combustion of biomass indigenous to South Asia, J. Geophys. Res.-Atmos., 108(D9), 4285, doi:10.1029/2002JD002981, 2003.

Sidhu, S., Gullett, B., Striebich, R., Klosterman, J., Contreras, J., and DeVito, M.: Endocrine disrupting chemical emissions from combustion sources: diesel particulate emissions and domestic waste open burn emissions, Atmos. Environ., 39, 801-811, 2005.

Simoneit, B. R. T., Cardoso, J. N., and Robinson, N.: An assessment of the origin and composition of higher molecular-weight organic-matter in aerosols over Amazonia, Chemosphere, 21, 1285-1301, 1990.

Simoneit, B. R. T., Sheng, G. Y., Chen, X. J., Fu, J. M., Zhang, J., and $\mathrm{Xu}, \mathrm{Y}$. P.: Molecular marker study of extractable organicmatter in aerosols from urban areas of China, Atmos. Environ., 25, 2111-2129, 1991.

Simoneit, B. R. T. and Elias, V. O.: Organic tracers from biomass burning in atmospheric particulate matter over the ocean, Mar. Chem., 69, 301-312, 2000.

Simoneit, B. R. T.: Biomass burning-a review of organic tracers for smoke from incomplete combustion, Appl. Geochem., 17, 129162, 2002.

Simoneit, B. R. T., Kobayashi, M., Mochida, M., Kawamura, K., Lee, M., Lim, H. J., Turpin, B. J., and Komazaki, Y.: Composition and major sources of organic compounds of aerosol particulate matter sampled during the ACE-Asia campaign, J. Geophys. Res.-Atmos., 109, D19S10, doi:10.1029/2004JD004598, 2004. 
Simoneit, B. R. T., Medeiros, P. M., and Didyk, B. M.: Combustion products of plastics as indicators for refuse burning in the atmosphere, Environ. Sci. Technol., 39, 6961-6970, 2005.

Stohl, A., Berg, T., Burkhart, J. F., Fjǽraa, A. M., Forster, C., Herber, A., Hov, Ø., Lunder, C., McMillan, W. W., Oltmans, S., Shiobara, M., Simpson, D., Solberg, S., Stebel, K., Ström, J., Tørseth, K., Treffeisen, R., Virkkunen, K., and Yttri, K. E.: Arctic smoke - record high air pollution levels in the European Arctic due to agricultural fires in Eastern Europe in spring 2006, Atmos. Chem. Phys., 7, 511-534, 2007,

http://www.atmos-chem-phys.net/7/511/2007/.

Stone, E. A., Lough, G. C., Schauer, J. J., Praveen, P. S., Corrigan, C. E., and Ramanathan, V.: Understanding the origin of black carbon in the atmospheric brown cloud over the Indian Ocean, J. Geophys. Res.-Atmos., 122, D22S23, doi:10.1029/2006JD008118, 2007.

Suh, I., Zhang, R., Molina, L. T., and Molina, M. J.: Oxidation mechanism of aromatic peroxy and bicyclic radicals from $\mathrm{OH}-$ toluene reactions, J. Am. Chem. Soc., 125, 12655-12665, 2003.

Surratt, J. D., Murphy, S. M., Kroll, J. H., Ng, N. L., Hildebrandt, L., Sorooshian, A., Szmigielski, R., Vermeylen, R., Maenhaut, W., Claeys, M., Flagan, R. C., and Seinfeld, J. H.: Chemical composition of secondary organic aerosol formed from the photooxidation of isoprene, J. Phys. Chem. A, 110, 9665-9690, 2006.

Szidat, S.: Sources of Asian Haze, Science, 323, 470-471, 2009.

Szmigielski, R., Surrat, J. D., González, G., Van der Veken, P., Kourtchev, I., Vermeylen, R., Blockhuys, F., Jaoui, M., Kleindienst, T. E., Lewandowski, M., Offenberg, J. H., Edney, E. O., Seinfeld, J. H., Maenhaut, W., and Claeys, M.: 3-Methyl1,2,3-butanetricarboxylic acid: An atmospheric tracer for terpene secondary organic aerosol, Geophys. Res. Lett., 34, L24811, doi:10.1029/2007GL031338, 2007.

van Pinxteren, D. and Herrmann, H.: Determination of functionalised carboxylic acids in atmospheric particles and cloud water using capillary electrophoresis/mass spectrometry, J. Chromatogr. A, 1171, 112-123, 2007.

Venkataraman, C., Habib, G., Eiguren-Fernandez, A., Miguel, A. H., and Friedlander, S. K.: Residential biofuels in South Asia: Carbonaceous aerosol emissions and climate impacts, Science, 307, 1454-1456, 2005.

Venkatesan, R., Rajendran, M., and Dasgupta, S.: Analysis and numerical simulation of the meteorological observations at a tropical coastal site Chennai in India, Atmos. Res., 92, 505-521, 2009.

Wan, E. C. H. and Yu, J. Z.: Analysis of sugars and sugar polyols in atmospheric aerosols by chloride attachment in Liquid Chromatography/Negative Ion Electrospray Mass Spectrometry, Environ. Sci. Technol., 41, 2459-2466, 2007.
Wang, G., Kawamura, K., Lee, S., Ho, K. F., and Cao, J. J.: Molecular, seasonal, and spatial distributions of organic aerosols from fourteen Chinese cities, Environ. Sci. Technol., 40, 4619-4625, 2006 .

Wang, G., Kawamura, K., and Lee, M.: Comparison of organic compositions in dust storm and normal aerosol samples collected at Gosan, Jeju Island, during spring 2005, Atmos. Environ., 43, 219-227, 2009.

Wang, H. B., Kawamura, K., and Shooter, D.: Wintertime organic aerosols in Christchurch and Auckland, New Zealand: Contributions of residential wood and coal burning and petroleum utilization, Environ. Sci. Technol., 40, 5257-5262, 2006 b.

Wang, W., Kourtchev, I., Graham, B., Cafmeyer, J., Maenhaut, W., and Claeys, M.: Characterization of oxygenated derivatives of isoprene related to 2-methyltetrols in Amazonian aerosols using trimethylsilylation and gas chromatography/ion trap mass spectrometry, Rapid Commun. Mass Sp., 19, 1343-1351, 2005.

Wang, W., Wu, M. H., Li, L., Zhang, T., Liu, X. D., Feng, J. L., Li, H. J., Wang, Y. J., Sheng, G. Y., Claeys, M., and Fu, J. M.: Polar organic tracers in $\mathrm{PM}_{2.5}$ aerosols from forests in eastern China, Atmos. Chem. Phys., 8, 7507-7518, 2008, http://www.atmos-chem-phys.net/8/7507/2008/.

Wolff, G. T. and Korsog, P. E.: Estimates of the contributions of sources to inhalable particulate concentrations in Detroit, Atmos. Environ., 19, 1399-1409, 1985.

Yan, B., Zheng, M., Hu, Y. T., Lee, S., Kim, H. K., and Russell, A. G.: Organic composition of carbonaceous aerosols in an aged prescribed fire plume, Atmos. Chem. Phys., 8, 6381-6394, 2008, http://www.atmos-chem-phys.net/8/6381/2008/.

Yassaa, N., Meklati, B. Y., Cecinato, A., and Marino, F.: Organic aerosols in urban and waste landfill of Algiers metropolitan area: Occurrence and sources, Environ. Sci. Technol., 35, 306-311, 2001.

Yttri, K. E., Dye, C., and Kiss, G.: Ambient aerosol concentrations of sugars and sugar-alcohols at four different sites in Norway, Atmos. Chem. Phys., 7, 4267-4279, 2007, http://www.atmos-chem-phys.net/7/4267/2007/.

Yu, J. Z., Cocker, D. R., Griffin, R. J., Flagan, R. C., and Seinfeld, J. H.: Gas-phase ozone oxidation products of monoterpenes: gaseous and particulate products, J. Atmos. Chem., 34, 207-258, 1999.

Zhang, R. Y., Suh, I., Zhao, J., Zhang, D., Fortner, E. C., Tie, X. X., Molina, L. T., and Molina, M. J.: Atmospheric new particle formation enhanced by organic acids, Science, 304, 1487-1490, 2004.

Zheng, M., Hagler, G. S. W., Ke, L., Bergin, M. H., Wang, F., Louie, P. K. K., Salmon, L., Sin, D. W. M., Yu, J. Z., and Schauer, J. J.: Composition and sources of carbonaceous aerosols at three contrasting sites in Hong Kong, J. Geophys. Res.-Atmos., 111, D20313, doi:10.1029/2006JD007074, 2006. 